\title{
SPITZER IRAC SPARSELY SAMPLED PHASE CURVE OF THE EXOPLANET WASP-14B
}

\author{
J. E. Krick ${ }^{1}$, J. Ingalls ${ }^{1}$, S. Carey ${ }^{1}$, K. von Braun ${ }^{2}$, S. R. Kane ${ }^{3}$, D. Ciardi ${ }^{1}$, P. Plavchan ${ }^{4}$, I. Wong ${ }^{5}$, and P. Lowrance ${ }^{1}$ \\ ${ }^{1}$ Spitzer Science Center, MS 314-6, California Institute of Technology, Jet Propulsion Laboratory, Pasadena, CA 91125, USA; jkrick@ caltech.edu \\ ${ }^{2}$ Lowell Observatory Flagstaff, AZ, USA \\ ${ }^{3}$ San Francisco State University San Francisco, CA, USA \\ ${ }^{4}$ Missouri State University Springfield, MO, USA \\ ${ }^{5}$ California Institute of Technology, Pasadena, CA, USA \\ Received 2015 September 4; accepted 2016 March 9; published 2016 June 7
}

\begin{abstract}
Motivated by a high Spitzer IRAC oversubscription rate, we present a new technique of randomly and sparsely sampling the phase curves of hot Jupiters. Snapshot phase curves are enabled by technical advances in precision pointing as well as careful characterization of a portion of the central pixel on the array. This method allows for observations which are a factor of approximately two more efficient than full phase curve observations, and are furthermore easier to insert into the Spitzer observing schedule. We present our pilot study from this program using the exoplanet WASP-14b. Data of this system were taken both as a sparsely sampled phase curve as well as a staring-mode phase curve. Both data sets, as well as snapshot-style observations of a calibration star, are used to validate this technique. By fitting our WASP-14b phase snapshot data set, we successfully recover physical parameters for the transit and eclipse depths as well as the amplitude and maximum and minimum of the phase curve shape of this slightly eccentric hot Jupiter. We place a limit on the potential phase to phase variation of these parameters since our data are taken over many phases over the course of a year. We see no evidence for eclipse depth variations compared to other published WASP-14b eclipse depths over a 3.5 year baseline.
\end{abstract}

Key words: instrumentation: detectors - methods: data analysis - planetary systems - planets and satellites: atmospheres - stars: individual (WASP-14, HD 158460)

\section{INTRODUCTION}

The study and characterization of exoplanetary atmospheres has been, and continues to be, one of the greatest legacies of the Spitzer Space Telescope (Fazio et al. 2004; Werner et al. 2004). The unique capabilities of Spitzer, coupled with the favorable planet-to-star contrast ratio in the infrared (Burrows et al. 2006), have allowed numerous achievements to date. For the first time, photons from a planet outside of our solar system were directly detected through observations of a planet's secondary eclipse, which gave us our first insight into the temperature of the planet's synchronously rotating dayside (e.g., Charbonneau et al. 2005; Deming et al. 2005). Emission spectrophotometry produced the first evidence of water molecules in dense exoplanetary atmospheres (e.g., Grillmair et al. 2008). The Infrared Array Camera (IRAC) high-precision photometry produced pioneering characterization of the atmospheres of extrasolar planets (e.g., Knutson et al. 2007a). While observations of secondary eclipses have been performed using ground-based facilities (e.g., Sing \& López-Morales 2009; Zhao et al. 2012), the study of the phase curves and the dynamics of exoplanetary atmospheres has only be achieved from space.

Coupled with theoretical modeling (e.g., Cooper \& Showman 2005; Burrows et al. 2006, 2008, 2010; Cowan \& Agol 2008, 2011; Lewis et al. 2010; Menou \& Rauscher 2010; Thrastarson \& Cho 2010) and Spitzer observations of secondary eclipses, phase curves can be used to place constraints on various parameters of exoplanet atmospheres, such as the atmospheric pressure structure, chemical composition, and the combination of albedo, different opacities, and the existence of a temperature inversion in the upper layers of the atmosphere.
A significant fraction of extrasolar planets whose atmospheres have been characterized to date are "hot Jupiters," i.e., planets approximately the size and mass of Jupiter that orbit their respective parent star once every few days. Their rotation rate is consequently believed to be synchronized with their orbital period, such that the same side of the planet always faces the star. These exoplanets receive approximately 10,000 to 100,000 times the flux received by Jupiter, which means that their dayside temperatures reach values as high as several thousand Kelvin while the nightside temperatures can be hundreds to thousands of Kelvin cooler. Additionally, hot Jupiters represent a simpler picture for circulation models in that they are in chemical equilibrium, largely cloud free, and no convection is expected in their atmospheres (Fortney et al. 2006). Understanding a sample of hot Jupiters is a vital stepping stone on the path to future characterization of cooler planets, including those in the habitable zones, by future NASA space missions such as the James Webb Space Telescope.

About a dozen infrared phase curves have been published to date (see review in Wong et al. 2015b; Harrington et al. 2006; Cowan et al. 2007, 2012; Knutson et al. 2007a, 2009b, 2009c, 2012; Laughlin et al. 2009; Crossfield et al. 2010, 2012a, 2012b; Demory et al. 2013; Lewis et al. 2013; Maxted et al. 2013; Zellem et al. 2014; Wong et al. 2015a, 2015b). The next scientific breakthrough in this field will come from exploring a large sample of extrasolar planets and determining how their atmospheric properties depend on physical conditions: comparative atmospheric sciences. The shape of the infrared phase curve can be interpreted as a longitudinal brightness temperature distribution across the planet and provides the best measurement of the efficiency of energy transport in the atmosphere. There are many factors involved in understanding the energy redistribution from the dayside to nightside (Burrows et al. 2006, 2008; Cowan \& Agol 2011). The 
measured energy emitted by the planet should equal the energy received by the planet from the star, modulo non-zero Bond albedo values and assuming no residual energy from planet formation (Knutson et al. 2009a). The longitudinal temperature distribution is driven by three effects: (1) the time it takes to absorb and reemit flux in the planet's atmosphere (radiative timescale), (2) the time it takes to transport a parcel of heated gas to the planet's nightside (advective timescale), and (3) chemistry which can effect the pressure level of the atmosphere probed at any wavelength. Any potential shift in time between the brightness maximum of the phase curve and the time of secondary eclipse (i.e., when the substellar spot is visible) is an indicator of the relative strength of the radiative (heating/ cooling) and advective (wind) processes. In addition, there are deviations in the planetary emission from a blackbody spectrum, such as inversion layers at the observed (wavelength-dependent) height in the atmosphere, which may affect the longitudinal temperature distribution.

The interpretation of published phase curves (e.g., Cowan \& Agol 2011; Perez-Becker \& Showman 2013; Schwartz \& Cowan 2015; Wong et al. 2015b) has clearly illustrated a diversity in atmospheric circulation patterns within the exoplanet population studied thus far. The reasons for this are not clear, principally due to a lack of data. We hope to add data to this effort, starting with this paper, which studies the phase curves of hot Jupiters through a novel snapshot technique that can help us to compile a large sample with less observing time invested on the highly oversubscribed Spitzer Space Telescope.

Two new techniques are presented in this paper: (1) using a gain map data set to remove intrapixel gain effect in the data and (2) sparsely sampled phase curves. This sort of project is made possible by the advent of precision pointing and characterization of the intrapixel sensitivity of the central pixel in the IRAC subarray. Phase curve observations do not have to be conducted in the time-consuming manner of multiple-day, consecutive observations. Instead, given that we know the periods of the planets, it is more efficient to build up phase curves over time from many, randomly spaced, short duration observations (see Section 2 for a more detailed description). The slew time of the Spitzer space telescope is relatively fast, and so there is no large penalty to observing targets with multiple epochs.

Something similar to our snapshot technique was used in Cowan et al. (2007). However, those observations nodded back and forth between the science target and a flux calibration star. This prevented them from making a correction for the intrapixel gain effect because the nods end up on different positions on the pixel. We have developed novel approaches, described below, in both observation planning and data reduction to correct for the intrapixel gain effect and other instrumental noise sources.

We present our pilot study with these new techniques of the hot Jupiter WASP-14b, a 7.3 Jupiter mass planet with an eccentricity of 0.083 (Wong et al. 2015b) orbiting an F5V star at a radius of 0.036 au with a 2.24 day period (Ehrenreich \& Désert 2011; Blecic et al. 2013). The star has a brightness of $K=8.6$ with $T_{\text {eff }}=6480 \pm 140 \mathrm{~K}$ and solar metallicity (Joshi et al. 2009). The above properties make this planet a hot Jupiter prototype. Joshi et al. (2009) note a density for this planet of $4.6 \mathrm{~g} \mathrm{~cm}^{-3}$, which is relatively high given that its radius is $1.26 R_{\text {Jupiter }}$. WASP-14b is known to have a significant spin- orbit misalignment, which, coupled with its eccentricity, is indicative of the orbital evolution of this massive planet (Johnson et al. 2009).

WASP-14b is predicted to have a thermal inversion based on its high level of irradiation and the activity of its parent star (Fortney et al. 2008; Knutson et al. 2010); however, Blecic et al. (2013) find that eclipse spectroscopy at 3.6, 4.5, and $8.0 \mu \mathrm{m}$ are well fit with no thermal inversion. Madhusudhan (2012) attribute the shape of the broadband eclipse spectrum to either possible condensation and gravitational settling of the $\mathrm{TiO}$ and VO (Spiegel et al. 2009), or a carbon-rich atmosphere with naturally low $\mathrm{TiO}$ and VO. Blecic et al. (2013) find evidence for a relatively low $(<30 \%)$ day-night energy redistribution and that the dayside spectrum of WASP-14b is consistent with both carbon-rich and oxygen-rich chemistry, with the latter being a marginally better fit.

This paper is structured in the following manner. Section 2 describes our strategy for snapshot observations including a discussion of stellar variability. Section 3 discusses systematic effects in IRAC data and our mitigation techniques. The data are described in Section 4 and our data reduction methods are explained in Section 5. The results and discussion are presented in Section 6, and the paper concludes in Section 7. Throughout the paper, we will refer interchangeably to "Ch1" or $3.6 \mu \mathrm{m}$ and "Ch2" or $4.5 \mu \mathrm{m}$.

\section{SNAPSHOT STRATEGY}

"Snapshot Phase Curves" is a program to observe a planethosting star for one epoch, and then later re-point to that star and observe it for another epoch, repeated at random intervals determined by brief holes in the Spitzer observing schedule, until we have built up a well-sampled phase curve. Building phase curves in this way keeps data volumes low and does not require the observatory to be taken over for days at a time, as is the case for traditional Spitzer exoplanet phase curve measurements. Snapshots are more efficient by a factor of two to three compared to long continuous staring mode observations, depending on the period, and are easier to schedule, which is especially important as the mission goes forward.

We set the duration of a single epoch snapshot at 30 minutes. This timescale is chosen for two reasons: (1) to stay within the roughly 30-40 minute pointing wobble (see Krick et al. 2015 for a discussion of spacecraft motions), and (2) it allows us to build up enough signal when binning to reach our signal-tonoise ratio $(\mathrm{S} / \mathrm{N})$ goals (described below).

We investigate the number of snapshot-style observations required to adequately sample a phase curve. We constructed a Monte Carlo simulation of phase curves and simulated data sets that assume a random distribution of 30 minute long snapshots (termed Astronomical Observation Requests (AORs) by Spitzer) in orbital phase-space. These simulated data sets are very simple approximations of IRAC photometry with a noise level commensurate with the expected IRAC noise for a specific brightness star (this simulation was made prior to the more detailed IRAC data simulator; Ingalls et al. 2016). The success of a simulation is judged by how well the phase amplitude measured on the simulated data set matches the real (input) amplitude. The assumptions that go into this include (1) an expected phase curve amplitude of $0.06 \%$ (the smallest amplitude predicted for known hot Jupiters at the time of observation planning in 2011), (2) a photometric uncertainty per data point of $0.02 \%$ in relative flux, (3) a sinusoidal shape 

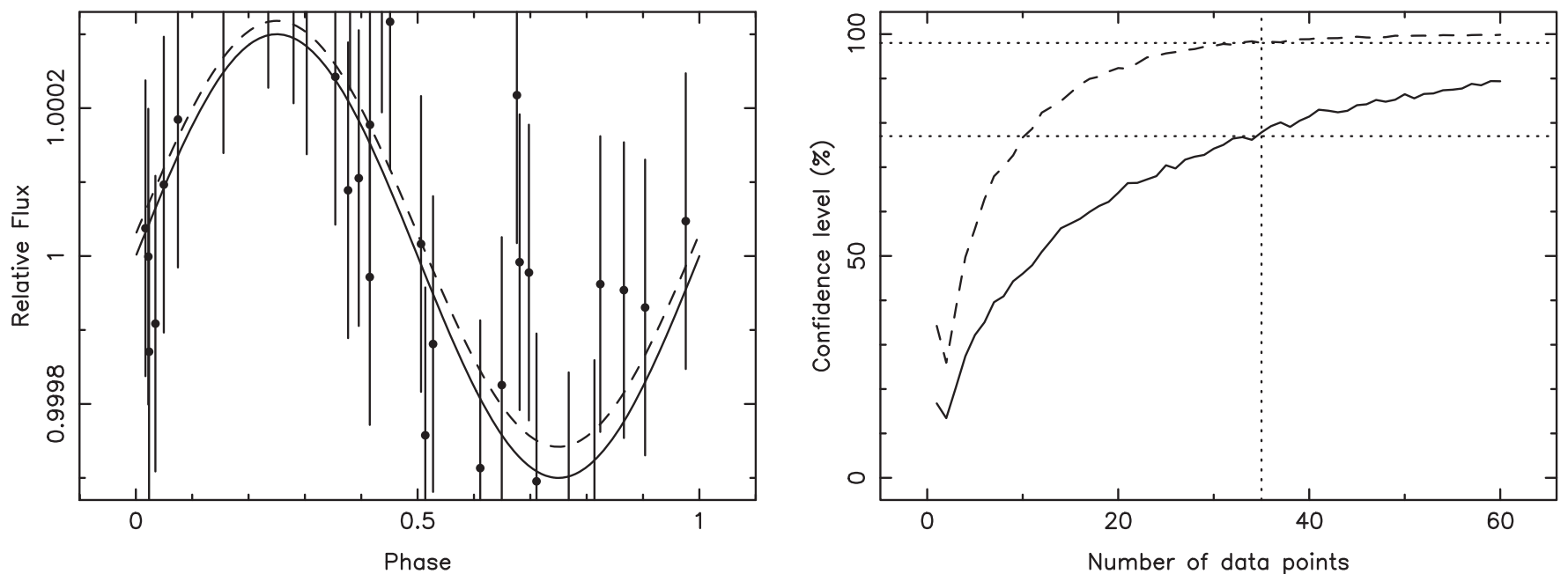

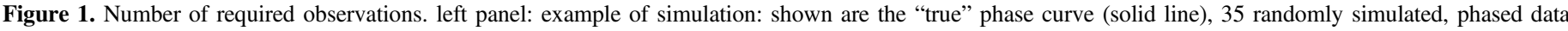

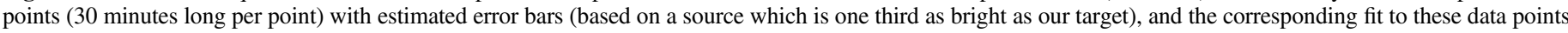

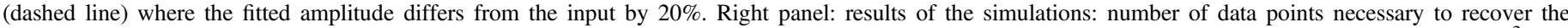

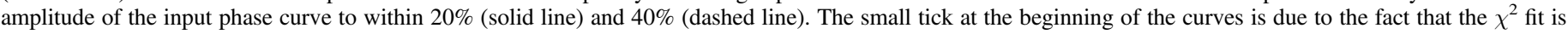

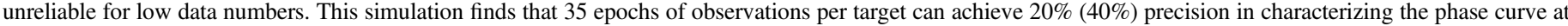
the $77 \%$ (99\%) confidence level for these faintest targets, and will do much better for brighter ones such as WASP-14b. See Section 2 for more details.

of the phase curve (Cowan et al. 2007), and (4) that the combined data for a given target cover a time-span much longer than the period of the exoplanet. Assumption (4), combined with a short AOR duration, ensures that the simulation results are independent of the planetary orbital duration. Assumption (2), that of photometric uncertainty, is based on signal-to-noise calculations for 30 minutes on a $25 \mathrm{mJy}$ source, which is a factor of three fainter than WASP-14b to accommodate all of the possible targets in our extended program.

In our simulation, the number of snapshot-style AORs was incremented from 1 to 60 . The top range of the number of simulated AORs represents a rough boundary where it might not be worth using this technique because the overall time required for snapshots would be similar to a continuous phase curve (depending on the period of the planet). We generated the Monte Carlo simulation with 5000 data sets, or sets of AORs, for each number of snapshot-style AOR bins. A $\chi^{2}$ fit was performed for each data set and the result was compared to the input parameters. We show the results in Figure 1. The left panel illustrates an example simulated data set of 35 measurements where the solid line is the model used to generate the data and the dashed line is the best fit to these data. The right panel summarizes all of the simulations. The dashed line represents those simulations where the $\chi^{2}$ fits produced an amplitude within $40 \%$ of the actual amplitude, constituting a marginal detection, but nevertheless providing a quantitative upper limit on the exoplanetary day/night contrast. The solid line indicates the percentage of data sets for which a measured amplitude within $20 \%$ of the actual amplitude was recovered. At this level of precision, much more quantitative constraints can be imposed on the atmospheric properties. Thus, for 35 measurements per target, we can recover the amplitude of the phase curve to within $20 \%$ of its input value $77 \%$ of the time, and to within $40 \%$ of the input value $99 \%$ of the time.

After observing 35 WASP-14b AORs in 2012 September, we noted that the assumed randomness of the observations was not achieved, and thus scheduled another 11 AORs in the hopes of filling in those phase regions which were not covered in the original 35 AORs. Forty-six total snapshots provide a well- sampled phase curve with fortuitous observations of one AOR during transit and two AORs during eclipse.

\subsection{Stellar Variability}

If the star varies, then a snapshot light curve might simply be capturing stellar variation instead of planet phase variations. The planetary variations are temporally well separated from stellar variations because the timescales of planetary orbits are rarely similar to the timescales of stellar rotations. The concern is that because we observe over many months and many phases, we may see an offset in fluxes from one snapshot to the next due to stellar variability. We chose WASP-14b because, statistically, its spectral type (F5V) indicates that it should be relatively quiescent based on the average variability of stars in the Kepler input catalog, the periodogram of the original survey data, and the measured radial velocity (RV) residuals.

Looking at a sample of F stars in the Kepler Input Catalog, Ciardi et al. (2011) find an average dispersion in 30 minute bins over 33 days of 0.1 mmag in the optical for F stars with Kepler mags in the range of WASP-14b $\left(K_{p}\right.$ estimated to be 9.7$)$. We expect the IR variation to be smaller than these average optical variations. Also, based on an emission spectrum, Knutson et al. (2010) find that WASP-14 is inactive.

Blecic et al. (2013) examine the periodogram of the original WASP light curve data to search for periodic signals as evidence of stellar activity. They find no significant (false alarm probability of less than 0.05 ) periodic signals in that data set, implying no measurable stellar variability.

Finally, stellar activity in the form of non-radial pulsations, or inhomogeneous convection or spots can cause RV variations that might show up in the RV surveys published to date (Santos et al. 2000). Joshi et al. (2009), in the original discovery paper, quote RV residuals of $10.1 \mathrm{~m} \mathrm{~s}^{-1}$. Wong et al. (2015b) find a residual of $12.3 \mathrm{~m} \mathrm{~s}^{-1}$ based partially on data from Knutson et al. (2014). This is in the expected range for this type of star, and is not high enough to suggest stellar activity ( $>40 \mathrm{~m} \mathrm{~s}^{-1}$; Paulson et al. 2004). 


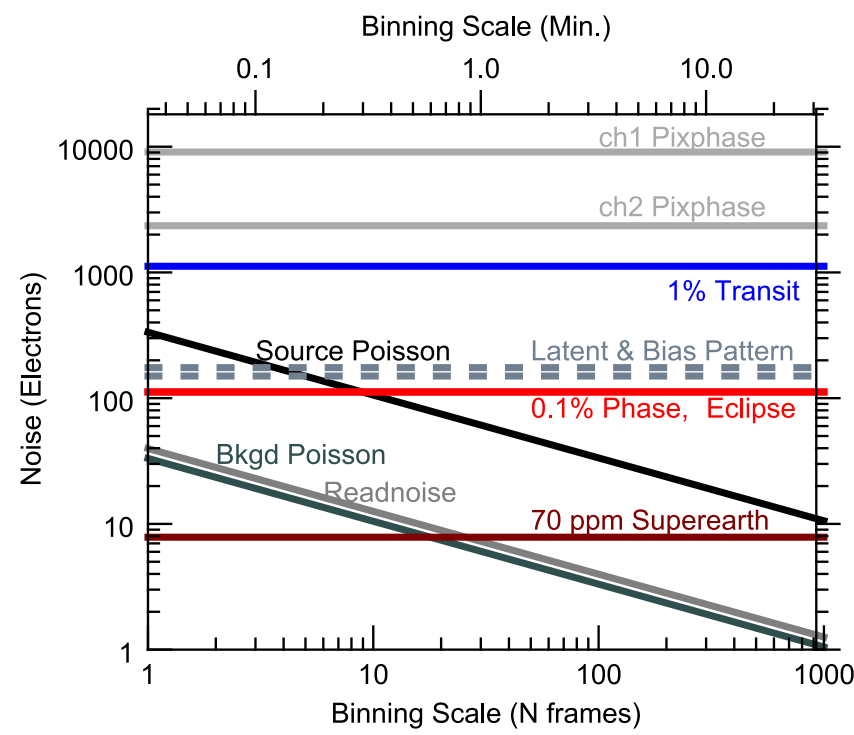

Figure 2. Noise sources in IRAC high-precision photometry. Black diagonal line shows the poisson noise due to a half-full well source. Noise due to that source's background and readnoise are shown in gray diagonal and are negligible for this work. The strength of the intrapixel gain effect is shown for both channels as horizontal light gray lines. Note that we choose to observe in $\mathrm{Ch} 2$ in large part because its intrapixel gain effect is lower than Ch1. Horizontal lines for $1 \%, 0.1 \%$, and $70 \mathrm{ppm}$ effects are shown for reference. Also noted as dashed lines are estimates for where latent and bias pattern noise will effect the photometry. See Section 3 for more details.

\section{IRAC SOURCES OF NOISE}

Data reduction for high-precision photometry is challenging due to both instrumental and astrophysical effects. Instrumental effects are discussed below and their relative strengths are shown in Figure 2. This figure includes the Poisson contribution from an assumed source at half-full well and the background along with the readnoise. The intrapixel gain effect for each channel is shown as a gray line and discussed in section Section 3.1. Because this is such a strong effect, it must be removed from the data before we can detect phase variations.

Estimates of the strength of the effect on photometry of latent images and a detector bias pattern are shown as dashed gray lines. Low-level persistent images exist after a bright star has been observed, and can last for many hours. These are discussed in Section 3.3. Changing bias patterns can be generated by a difference in the delay times before images are recorded from the darks to the science frames. The bias pattern effect has not been fully characterized and cannot be derived from this data set. The estimates of the bias effect used in this plot are determined from a set of five 5-10 hr continuous staring mode archival observations of a blank field (no star targeted). The level of this bias effect is uncertain and will vary as a function of time over the days to months between observations presented here.

As examples of the rough level of the signals relevant to exoplanet studies with IRAC, we also show the relative levels of a $1 \%$ transit depth, a $0.1 \%$ eclipse depth, and a $70 \mathrm{ppm}$ postulated Super-Earth secondary eclipse depth.

\subsection{Intrapixel Sensitivity}

Due to the under-sampled nature of the PSF, the warm IRAC arrays show variations of as much as $8 \%$ in sensitivity as the center of the PSF moves across a pixel due to normal spacecraft motions (Ingalls et al. 2012). These intrapixel gain variations are the largest source of correlated noise in IRAC photometry (see Figure 2). Many data reduction techniques rely on the science data themselves to remove the gain variations as a function of position (Charbonneau et al. 2005; Ballard et al. 2010; Knutson et al. 2012; Stevenson et al. 2012; Lewis et al. 2013; Deming et al. 2015). The limitation of selfcalibration techniques is that they do not work well for sparsely sampled data sets (many full phase curves). The SSC has generated a high-resolution gain map from standard star data (Ingalls et al. 2012) which can be used as an alternative when reducing data.

The use of the gain map reduction technique is reliant on Pointing Calibration Reference Sensor (PCRS) Peak-Up. PCRS Peak-Up uses the Spitzer PCRS to repeatedly position a target to within 0.25 IRAC pixels of an area of minimal gain variation. It is important to land on this "sweet spot" ([15.120,15.085] in ch2) because it (a) minimizes the effect on the photometry of standard telescope motions, (b) it is the most well-calibrated position on the detector, and (c) it enhances measurement repeatability from one epoch to the next. The minimization of the intrapixel gain effect happens both because that region is well calibrated and because the slope of the gain map is shallowest at that position. The change in gain as a function of position is minimized to $\sim 0.2 \%$ across the sweet spot, which is smaller than at other positions on the pixel.

Most staring mode exoplanet observations employ the SSCrecommended practice of observing 30 minutes pre-AOR to allow the telescope to settle. In the interest of time, we do not observe this pre-AOR, and instead peak-up directly to our target and start the snapshot observations.

The gain map ("pmap") data set uses the IRAC calibration stars KF09T1 in ch1 and BD+67 1044 in ch2 taken with subarray 0.4 and $0.1 \mathrm{~s}$ frame times, respectively. The 2015 February 26 version of the $4.5 \mu \mathrm{m}$ pmap data set used in this reduction includes a total of 409,539 photometry points, $90 \%$ of which are within the sweet spot. Initial mapping of the central pixel deliberately included the whole pixel as work was beginning to define the sweet spot. In general, PCRS Peak-Up places a target within the sweet spot $98 \%$ of the time (Ingalls et al. 2012). This paper only uses ch2. All of the gain map data are reduced in the same manner as the science observations.

The limitation of using a gain map for removing gain variations from the data is that it cannot be applied to data with positions off the sweet spot. This is more often the case for targets which are faint or extremely bright, and for high proper motion stars. We leave a comparison of the various data reduction and analysis techniques to a future study.

\subsection{Photometric Stability}

The snapshot technique only works if aperture photometry is stable as a function of time on year-long timescales. This is because both the gain map calibration data set and the observations of WASP-14b were taken over many years. The gain map calibration data set (see Section 3.1) was observed starting in 2011, with additional data taken as recently as winter 2014 , and the data on WASP-14b was taken over a 1.5 year baseline (see Section 4.) The calibration data set needs to be both consistent over the years it was observed and capable of correcting data taken at any time during the mission. 


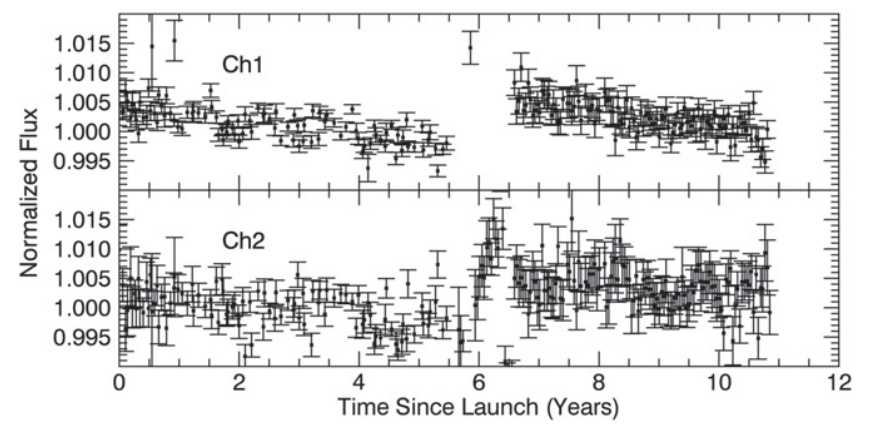

Figure 3. Photometric stability as a function of time: $3.6 \mu \mathrm{m}$ on the top and $4.5 \mu \mathrm{m}$ on the bottom. This plot shows aperture photometry of the ensemble of calibration stars over the entire mission to date. There is a slight degradation as a function of time which is possibly due to radiation damage to the optics. The gap around six years is the transition from cryo to warm where for a short time we used a different calibration which will effect the aperture fluxes. See Section 3.2 for more details.

To understand the photometric stability of the IRAC detectors as a function of time, we examined the existing calibration data taken over the course of the entire 11 year mission to date. Figure 3 shows aperture photometry of seven IRAC primary calibration stars binned together on two week timescales. Primary calibrators are described in detail in Reach et al. (2005). Their main function is to determine the absolute calibration of the instrument.

There is a statistically significant decrease in the sensitivity of both ch1 and ch2 over the course of the mission. The degradation is extremely small, of the order of $0.1 \%$ per year in ch1 and $0.05 \%$ per year in ch2. The decrease in sensitivity is potentially caused by radiation damage to the optics. Individual light curves for each of the calibration stars used in this analysis were checked to rule out the hypothesis that one or two of the stars varied in a way so as to be the sole cause of the measured decrease. While the slope for each individual star is not as well measured as for the ensemble of stars, it is apparent that the decreasing trend is not caused by outliers. We also rule out the solar cycle as the cause of flux degradation by examining the cosmic-ray rate as a function of time throughout the mission.

In light of this very small flux degradation, we have corrected the photometry of both the gain map data set and the exoplanet host stars by a linear function in time which decreases at $0.05 \%$ per year.

\subsection{Persistent Images}

Both IRAC channels sometimes show residual images of a source after it has been moved off of a pixel. When a pixel is illuminated, a small fraction of the photoelectrons are trapped. The traps have characteristic decay rates and can release a hole or electron that accumulates on the integrating node long after the illumination has ceased. Persistent images on the IRAC array start out as positive flux remaining after a bright source has been observed. At some later time, the residual images turn from positive to negative, so that they are actually below the background level (trapping a hole instead of trapping an electron). Positive and negative persistent images in either the aperture or background annulus in either the dark frame or the science frames can lead to artificial increases or decreases in the aperture photometry fluxes. We will use the terms persistent images and latents interchangeably throughout the discussion.
We have learned from this work that persistent images are pervasive. Short-term persistent images start at about $1 \%$ of the source flux and can be seen to decay over the course of minutes to hours. We look for long-term latents by median combining all of the warm mission darks at the $2 \mathrm{~s}$ frame time into a "superdark." We also make yearly and seasonal superdarks which are a median combination of a single year's worth of darks or a single season's (e.g., January-March) worth of darks throughout the mission. Differencing the mission-long superdark from the yearly and seasonal darks reveals low-level, long-term latent patterns which change from year to year and season to season. These residual latent images are either caused by observing cadence (coincidentally, Spitzer uses more subarray in the fall season) or by long-lasting, low-level, persistent images. Beyond knowing of their existence, it is very difficult to track these low-level latent images post facto, especially since we know that the latents are both growing with new observations dependent on the specific observing history, and shrinking over time as they dissipate.

Long-term latents are significant for this work because they change on the timescales over which our observations are made. The effect on snapshot photometry will have a random component, as the scheduling of the snapshots was random. Our superdark analysis shows that persistent images can effect the photometry of snapshots at the $0.1 \%$ level (see Figure 2). In order to remove this effect for future snapshot phase curve observations, we recommend observing a dither pattern on a blank region before and/or after each snapshot. This will ultimately provide a dark observation that is specific for each snapshot observation. The choice of observing the dither before and after will depend on whether or not the persistent image is stable on 30 minute timescales, of which we do not have a good understanding. Adding these dithered observations will increase the time required for these types of sparsely sampled phase curves. If the persistent image level were changing significantly on the 30 minute timescale of the snapshots, then we might expect to see its signature in the rms versus Binning plots, which instead show only Poisson noise, see Section 6.3.1.

This latent in the darks is not seen in the instrument stability section (Section 3.2) where tests were performed on photometry of calibration stars because those observations are dithered around in position, as opposed to holding the observatory in one single position.

\section{Spitzer WARM IRAC OBSERVATIONS}

Here, we present snapshot observations of a pilot set of observations of WASP-14b, which can be extended to more exoplanetary systems. In addition, we also discuss archival continuous staring mode observations of WASP-14 and snapshot observations of the calibration star HD 158460 for verification of the observing strategy. Observing parameters are listed in Table 1. All of the observations discussed in this paper were taken with Warm IRAC in staring mode at $4.5 \mu \mathrm{m}$ with the target observed as close as possible to the sweet spot of the central pixel of the subarray.

We choose $\mathrm{Ch} 2$, and not $\mathrm{Ch} 1$, for both scientific and technical reasons. First, the predicted planet-to-star flux ratio values are larger at $4.5 \mu \mathrm{m}$ (by tens of percent), which increases the planetary contribution to the photometry. Second, Ch2 is overall a better behaved instrument in the warm mission (see Figure 2). In particular, the pixel phase effect is a smaller effect 
Table 1

Summary of Observations

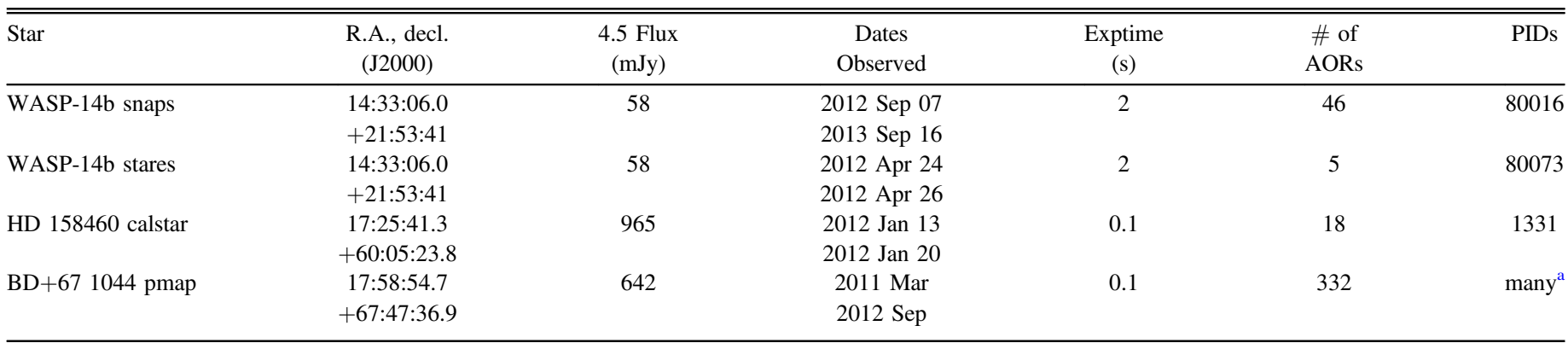

Note.

${ }^{\mathrm{a}} 1320,1326,1328,1331,1333,1336,1338,1346,1658,1659,1669$.

in Ch2 than Ch1 (Ingalls et al. 2012). The only minor disadvantage to choosing $\mathrm{Ch} 2$ of which we are aware is that the stars are fainter.

In Staring mode, no dithering or mapping is used; the telescope is not intentionally moved after it arrives on target. The subarray is a $32 \times 32$ pixel portion of the full detector array. Images are stored in sets of 64 subframes, tied together into one FITS file. Data described in this paper are from all archival data on Program IDs (PIDs) 80016, 80073, and calibration PIDs 1320, 1326, 1328, 1331, 1333, 1336, 1338, 1346, 1658, 1659, 1669.

PCRS peak-up on the target was used prior to all AORs to reliably put the target star on the sweet spot, which is necessary for our reduction technique. We have only mapped the gain variations to sufficient precision for this work over the single sweet spot of the central subarray pixel in both channels (see Section 3.1). If the target does not land on the sweet spot where the existing gain map data set can provide calibration of the intrapixel gain effect, then this technique is not possible and snapshots cannot be used to build phase curves.

For WASP-14b, a two second frame time was chosen from among the fixed set available to observe this $68 \mathrm{mJy}$ star at $\sim 38 \%$ of full well where the IRAC detectors have the least nonlinearity (see Section 5.4). In total, 46 snapshot visits were observed randomly throughout two visibility windows separated by one year. The first 35 observations were taken from 2012 September 07 to 2012 September 27 UTC. A further 11 snapshots were observed from 2013 August 24 to 2013 September 16 UTC. Each snapshot's AOR produced 14 subarray FITS files containing 896 individual images. Continuous staring mode observations were made as 5 roughly $12 \mathrm{hr}$ long consecutive AORs from 2012 April 24 to 2012 April 26 UTC.

For HD 158460, a $0.1 \mathrm{~s}$ frame time was chosen to observe this $1187 \mathrm{mJy}$ calibration star at $\sim 32 \%$ of full well where the IRAC detectors have the least nonlinearities. In total, 18 snapshot observations were taken randomly from 2012 January 13 through 2012 January 20 UTC. Each snapshot AOR produced 210 subarray FITS files containing 13,440 individual photometry measurements.

We do not use the standard 30 minute pre-AOR because that would remove the efficiency gain provided by using snapshots over continuous stares. The goal of that pre-AOR is to allow the telescope motion to settle upon first arriving on the target so that the target does not drift off of the sweet spot. Since our observations are only 30 minutes long, as opposed to the manyhour continuous stares, the drift in position that occurs within the observations keeps the majority of the snapshots within the calibrated region of the pixel, and so the pre-AOR is not necessary for this particular application.

Four of the 51 AORs did not end up with at least $20 \%$ of their data points on the sweet spot. This includes one continuous staring mode AOR (containing the second observation of the secondary eclipse) and three of the snapshot AORs. These non-sweet-spot AORs are ignored in the following analysis.

\section{DATA REDUCTION}

Data reduction is exactly the same for all of the snapshot and continuous staring mode data sets. We start our data reduction from the Basic Calibrated Data (BCD) files provided by the SSC data pipeline, software version S19.1.0. The pipeline applies a dark subtraction, linearization, flat-field correction, and conversion to flux units. The first subframe of each 64frame BCD is removed from this analysis due to its higher bias level. The higher bias level is caused by the larger elapsed time between BCDs as opposed to consecutive subframes within a single BCD. This effect is referred to as the "first frame effect" in the IRAC documentation (http://irsa.ipac.caltech.edu/data/ SPITZER/docs/irac/iracinstrumenthandbook/).

\subsection{Superdark}

Dark current in all IRAC frames is measured with a shutterless system designed by the IRAC instrument team (described in detail in Krick et al. 2009). In summary, weekly observations at each frame time for both the sub- and full-array are made of a low background field at the north ecliptic pole. These images are then vetted for obvious persistent images (latents), and median combined to make a weekly dark. For each data frame, the nearest-in-time dark is used by the pipeline to correct that frame. This means that for our data sets which span multiple weeks/years, different dark frames are subtracted from different AORs. For our science goals, we need to put aperture photometry from the whole set of snapshot observations on a single flux level. To determine how best to do this, we examine the effect of using weekly darks in comparison to a mission-long superdark. The reason for concern is that a latent image in the dark frame near the center of the frame (either in the aperture or the background annulus) will cause changes in the aperture photometry of the target from one AOR to the next.

Because the latent population changes on day-to-week-long timescales, the advantage of weekly darks is that they 
potentially have the same latent in them that the science data has, allowing us to remove latents from the science frames. However, it is also possible that the weekly darks have latents in them which have faded by the time the science data are observed, thereby adding a source of noise to the science data. It is possible to generate a superdark from archival data by median combining all of the dark images taken during the entire duration of the warm mission to date. Each frame time gets its own superdark. The advantage of superdarks is that they have no latent structure in them. The disadvantage of superdarks is that they cannot remove latents which are present in the science data.

When making both the weekly and superdarks, the median combine will reject all astronomical sources. Aside from latent images, we expect that the only other difference between the superdark and weekly dark to be a change in the background level due to the zodiacal light component varies as a function of time (Krick et al. 2012). However, since we are performing aperture photometry, we do not care that the mean level of the dark is incorrect by the amount that the zodiacal light fluctuates over a one-year baseline. Throughout the warm mission, we do not see evidence of a change in the background pattern in the darks. The superdark is applied to each data frame by first backing out the pipeline calibrations already applied to the BCD including the weekly dark, and then calibrating those images with our superdark. All of the calibration files required to do this are provided by the SSC in the Spitzer Heritage Archive.

Without the luxury of darks taken directly adjacent to the snapshots, we are forced to choose between using the weekly darks and the superdarks. We use the standard deviation within data sets as the metric for the decision of weekly darks versus superdarks. We find the lowest scatter photometry using weekly darks for the gain map calibration data set and superdarks for the WASP-14b data set. This is probably due to the persistent images we see in the snapshot data set (see Section 6.1.1), whereas the gain map data set is so much larger that the individual frames have not been studied in such detail.

\subsection{Centroiding and Aperture Photometry}

The star centers on each subframe are determined using the SSC provided box_centroider.pro (http://irachpp.spitzer. caltech.edu/page/contrib). This code uses an iterative process with the first moment of light to find the star centers. We choose this technique because it is simple, robust, repeatable, and easy to code for comparison with other groups using different methods. We have not tested other centroiding methods on this data set. For WASP-14b, there is a faint star $\sim 11.4$ arcsec from the center of WASP-14 (not cataloged in Simbad so we do not have literature fluxes for it). A $6 \times 6$ arcsec region around the right ascension (R.A.) and declination (decl.) of that star is masked in all images to block the light coming from that star before centroiding and performing photometry. The masked region is well outside of the photometric aperture, but does cover part of the background annulus.

Aperture size is chosen for this data set by finding the aperture radius that maximizes the $\mathrm{S} / \mathrm{N}$ of the final reduced data set. We test eight apertures in 0.25 pixel bins from 1.5 to 3.25 pixels. For WASP-14b, a 2.25 pixel aperture minimizes the contribution from background noise, while including enough of the star's flux to maximize the $\mathrm{S} / \mathrm{N}$. Since the calibration star HD 158460 is a test data set, we hold the aperture size constant at 2.25 pixels for that data set as well. We use this same aperture size on the gain map data set for consistency.

A background annulus of 3-7 pixels (3!"6-8". 4) is used. We choose this annulus size for WASP-14b because there is a neighboring object in the images at about 10 pixels from the center of WASP-14; since we have no information on the variability of that source, we would like to exclude it from our background region as much as possible. Also, since we need to use a single, uniform method for finding the background on our target as well as on the very large gain map data set, we have chosen to stick with the 3-7 pixel annulus for the entire paper.

Along with centers and aperture fluxes, we calculate the number of noise pixels for each subframe as well as the individual components of the noise pixel in the $X$ and $Y$ direction, calculated using the SSC provided box_centroider. pro. The noise pixel parameter gives an indication of apparent size of the target star and is defined in the IRAC Instrument Handbook (http://irsa.ipac.caltech.edu/data/SPITZER/docs/ irac/iracinstrumenthandbook/5/) and in Mighell (2005) Lewis et al. (2013). Specifically, it is the equivalent number of pixels whose noise contributes to the flux of a point source. There is no evidence that the PSF itself is changing with time. However, at frequencies higher than the observation rate, oscillations of the spacecraft will have the effect of smearing out the image, thereby increasing the noise pixel without changing the centroids significantly.

For binning, we remove both position and flux outliers. We first remove points from our data set that are three standard deviations away from the global mean $Y$ position or 2.5 standard deviations away from the global mean $X$ position. The larger tolerance in the $Y$ direction is due to more native spacecraft motion in the $Y$ direction. We have checked individually many of these position outliers, and every one we have followed up is caused by a cosmic ray near the star which corrupts the measured positions. For both the snapshot and continuous staring data sets, we reject on average $\sim 1 \%$ of the data as position outliers. Flux outliers are removed using a running $3 \sigma$ mean.

Figure 4 shows the raw values of the $X$ centroid, $Y$ centroid, $X$ and $Y$ FWHM (second moment of intensity distribution), noise pixels, normalized background value, and normalized raw flux as a function of both orbital phase and time. The time plot includes a discontinuity of about a year between sets of observations. We also show a zoom-in for a few AORs on Figure 5. Figure 6 shows all of the snapshot AOR centroid positions over-plotted on an image of the intrapixel gain map to provide a sense of how accurate and precise the pointing is for this program. The color coding of the AORs remains consistent throughout all of the figures for this paper.

\subsection{Kernel Regression Gain Map Correction}

Data for both the snaps and stares were reduced using a Nadaraya-Watson-type kernel regression technique (Watson 1964; Nadaraya 1964). Kernel regression has been used extensively in the literature as a way of correcting for the IRAC intrapixel gain effect based on neighboring photometry points in the data set itself (often referred to as pixel mapping; Ballard et al. 2010; Knutson et al. 2012; Lewis et al. 2013; Zellem et al. 2014; Wong et al. 2015b). What is different from the literature methods, however, is that we use the photometry 

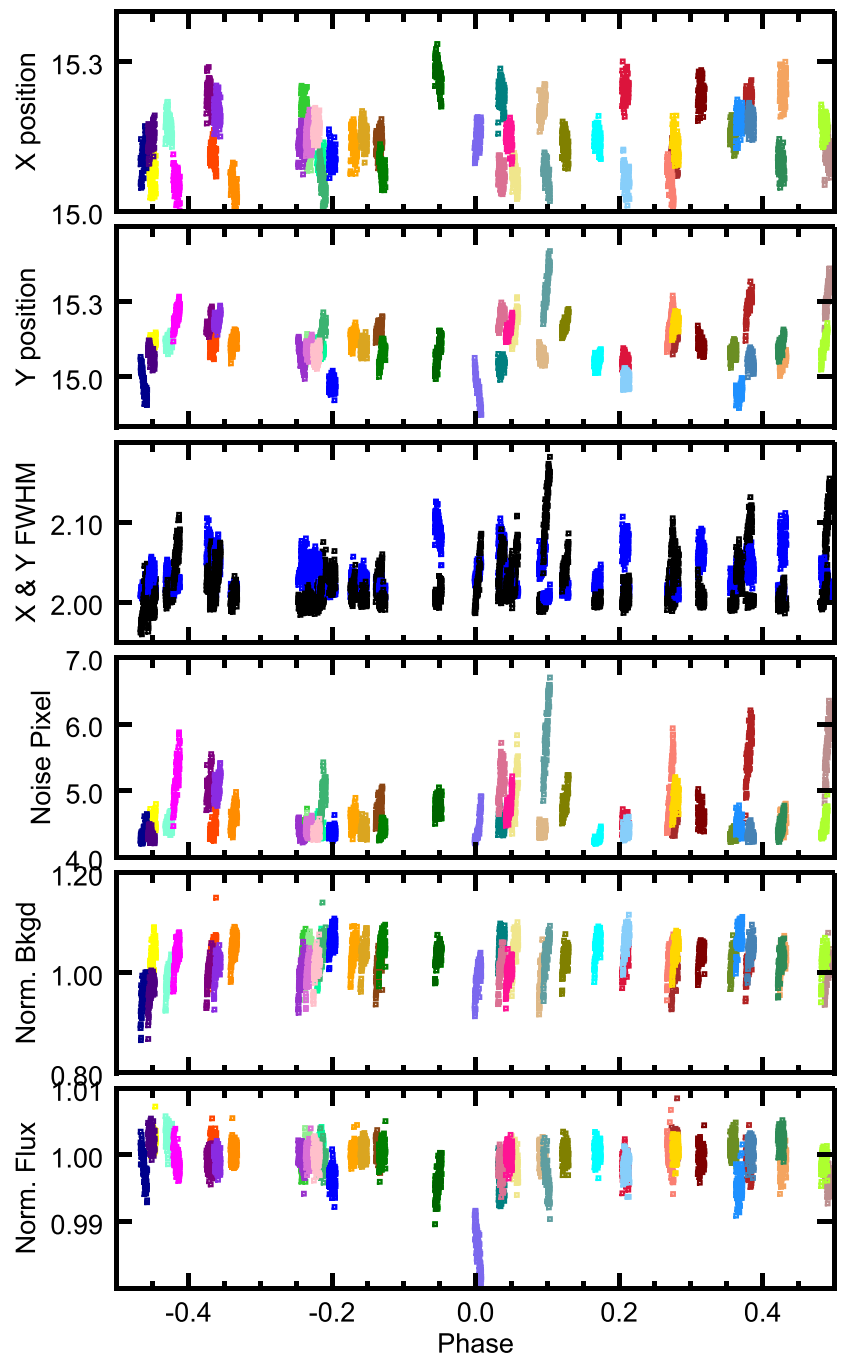

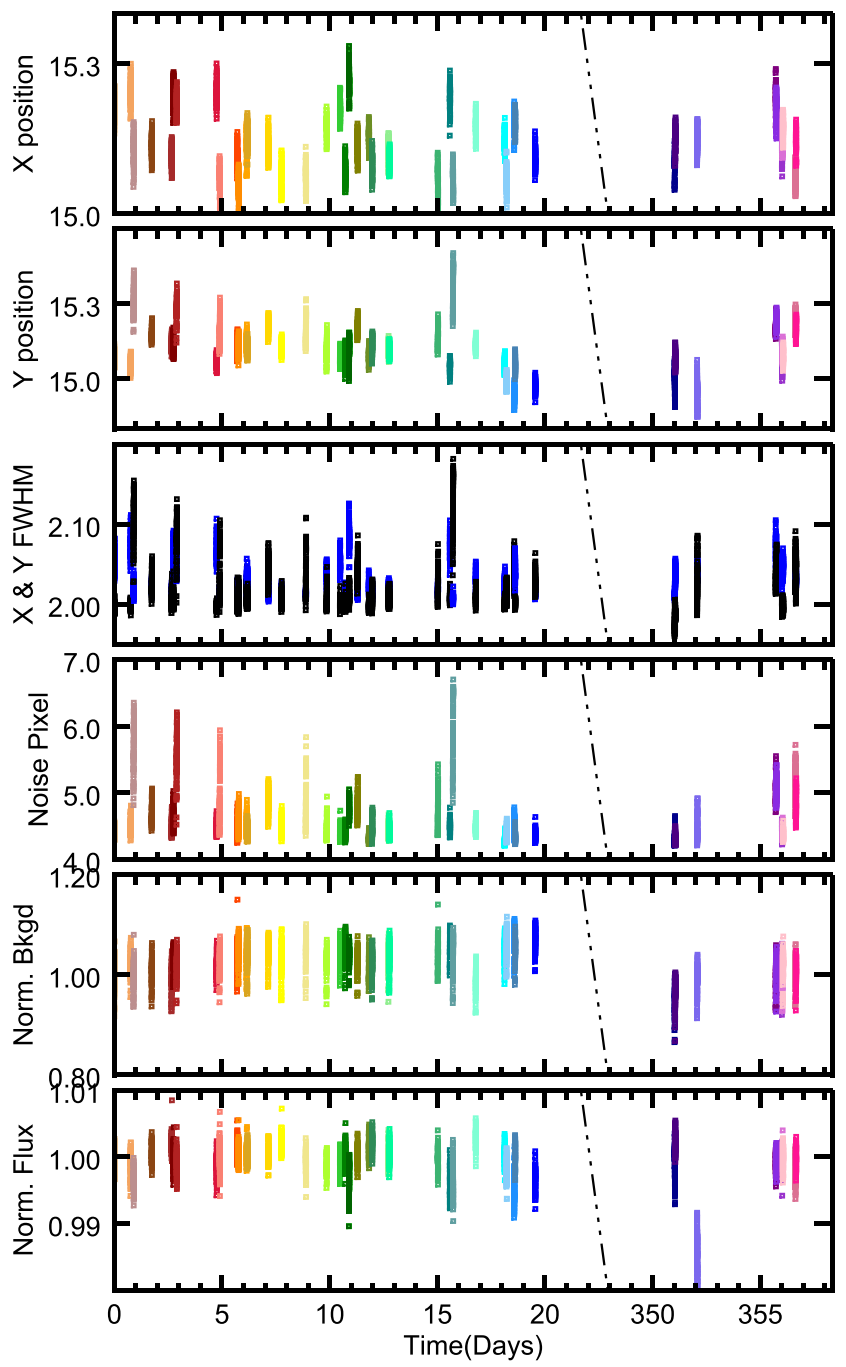

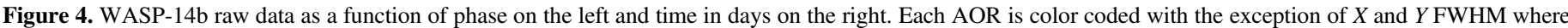

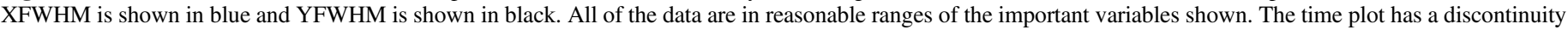
of about a year between the first 35 and the last 11 AORs shown by the diagonal dot-dashed lines. See Section 5.2 for more details.

of a separate calibration star BD+67 1044, which is not known to vary. As mentioned earlier, the Spitzer Science Center has accumulated approximately 400,000 measurements of this gain map calibration star, positioned all within 0.3 arcsec of the "sweet spot" (peak of response) of the ch2 subarray central pixel.

Kernel regression correction of science data begins by finding the $N$ nearest neighbors to a given target data point in the gain map calibration data set based on the Euclidean distance in $X$ and $Y$ centroid and noise pixels. The $N$ gain map points are weighted by a kernel which is a Gaussian function of the distance to the science data point. The width of the Gaussian kernels are computed on the fly as the coordinate standard deviations (in $x, y, \mathrm{NP}$, etc.) of the 50 neighbors in the pmap data set. The weighted kernels are then summed and normalized by the calibration star flux. The result is a prediction of the relative strength of the intrapixel gain map at the location of the science data point. To correct for the intrapixel gain we divided the science flux by this prediction.

The technique includes tunable parameters for the number of nearest neighbors $N$, the maximum distance from the science data point, and the minimum "occupation" number, a kernel- weighted measure of how many of the nearest neighbor gain map data points contributed to the result (see Ingalls et al. 2012 for details on the occupation). For the current program, we set the number of nearest neighbors to 50 (Lewis et al. 2013), the minimum distance to 0.0025 pixels, and the occupation number to 20 . This tight requirement on the proximity and quantity of gain map data helps to minimize the effects of persistent images that may exist in the pmap data set. As mentioned above, we did not include three AORs in our final analysis that have less than $20 \%$ of their photometry points within the sweet spot. One important difference between this method and literature kernel regression techniques for exoplanet reduction is that we did not build a correction from the science measurements themselves, and therefore minimize the risk of removing astrophysical signal.

An earlier version of this technique contained an intermediate step of computing the gain map on a regular grid in $X$ and $Y$ centroid and then interpolating to the science data positions (Ingalls et al. 2012). We find the direct nearest neighbors approach to be more effective (albeit more CPUintensive) because working from an intermediately gridded map introduces additional uncertainties inherent to gridding 


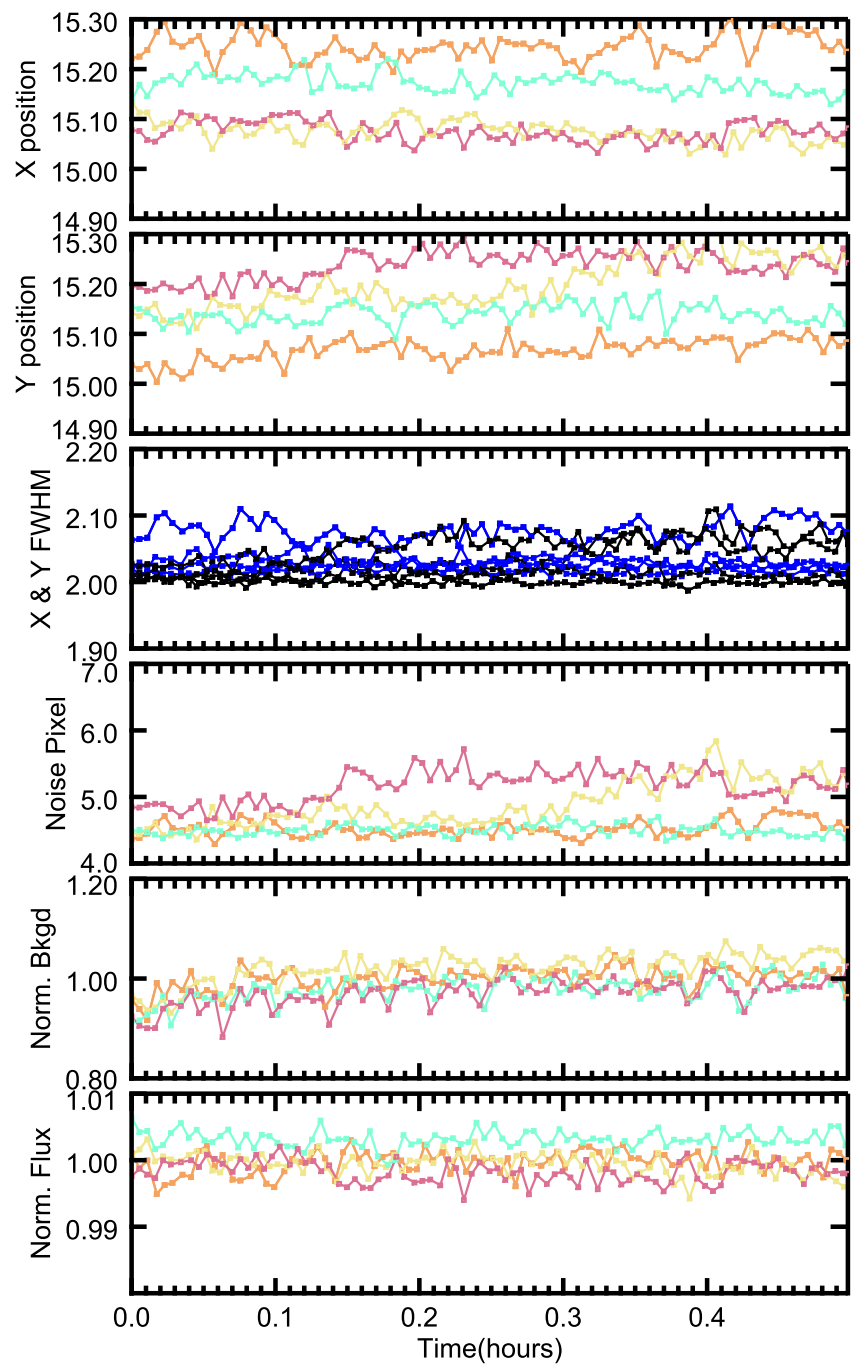

Figure 5. Zoom-in on WASP-14b raw data as a function of time for four random AORs spread throughout the set of observations. Each AOR is color coded with the exception of $X$ and $Y$ FWHM where XFWHM is shown in blue and YFWHM is shown in black. See Section 5.2 for more details.

and interpolation. The current method will be described more fully in a future paper (J. G. Ingalls et al. 2016, in preparation).

Figure 7 shows the final reduction of WASP-14b snapshots and continuous staring mode AORs phased to the literature period of 2.24376507 days (Wong et al. 2015b). Figure 8 shows the same data as a function of time instead of phase. Since there is about one year between each set of observations, we show this in two frames with the first set of 35 AORs in one frame, followed by the second set of 11 AORs in the other.

\subsection{Nonlinearity}

The IRAC detectors have a known nonlinearity such that a linear increase in the number of incoming photons does not lead to a linear increase in the flux measured on the detectors. The pipeline corrects for this nonlinearity to an accuracy of about $1 \%$. High-precision photometry studies that want to use the gain map taken at one flux level to correct science data taken potentially at another flux level are sensitive to a "residual nonlinearity" $(<1 \%)$. We have examined this residual nonlinearity in detail (Krick et al. 2015) and conclude that it does not effect flux as a function of position on the pixel for

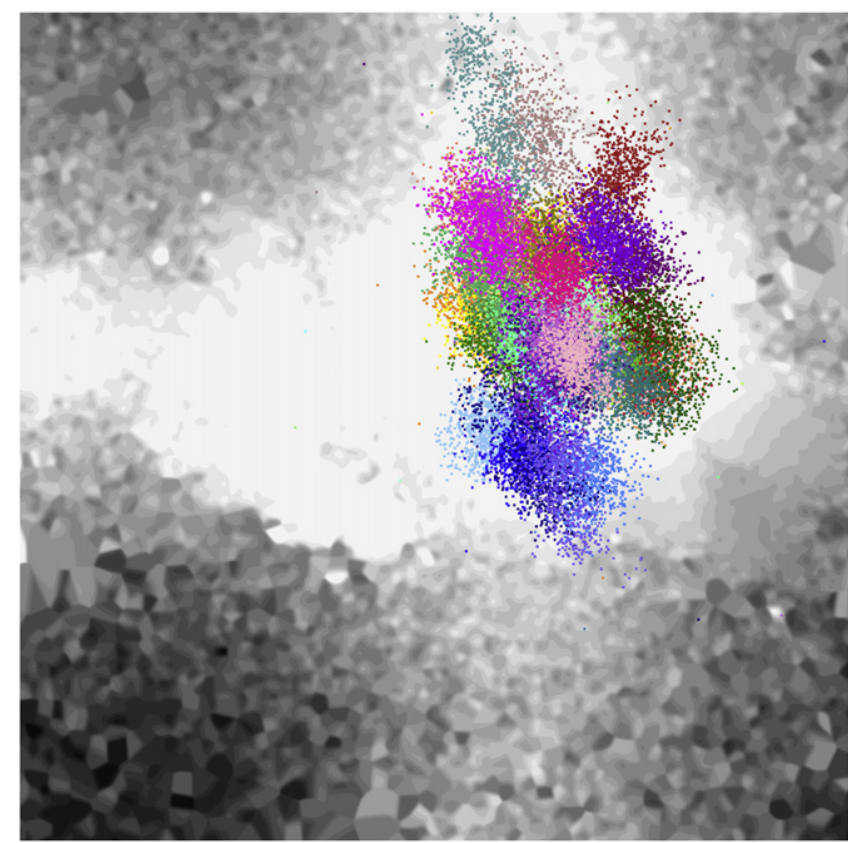

Figure 6. Grayscale image of the gain variations within a single pixel at the center of the subarray. The centroid positions of WASP-14b snapshot AORs are shown in the same color as the other WASP-14b figures. See Section 5.2 for more details.

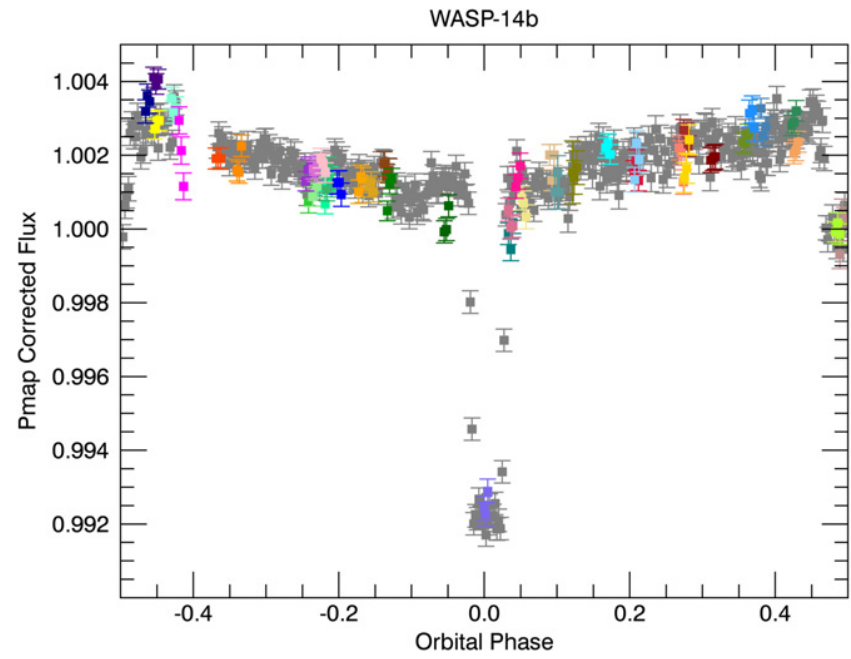

Figure 7. Final photometry of WASP-14b. Gray points show our reduction of the continuous staring mode data. Colored points show the snapshot data. All have been binned on the same timescale and normalized to the level in secondary eclipse. Zoom-ins on the transit and eclipse are shown in Figure 14. See Section 5.3 for more details.

observations with peak pixel counts between 1000 and 15,000 $\mathrm{DN}$ in ch2, which is the case for all of the observations here. WASP-14b has between 9000 and 10,000 DN in the peak pixel depending on the position.

\subsection{Electronic Ramp}

Some authors report an electronic ramp in flux measurements as a function of time in their warm IRAC data sets (Campo et al. 2011; Deming et al. 2011; Todorov et al. 2012, 2013). Ramps seen in the 5.8 and $8.0 \mu \mathrm{m}$ cryogenic data are probably not relevant because those are $\mathrm{Si}$ :As detectors, whereas the ch1 and 2 detectors are $\operatorname{InSb}$ (see http://irsa. 

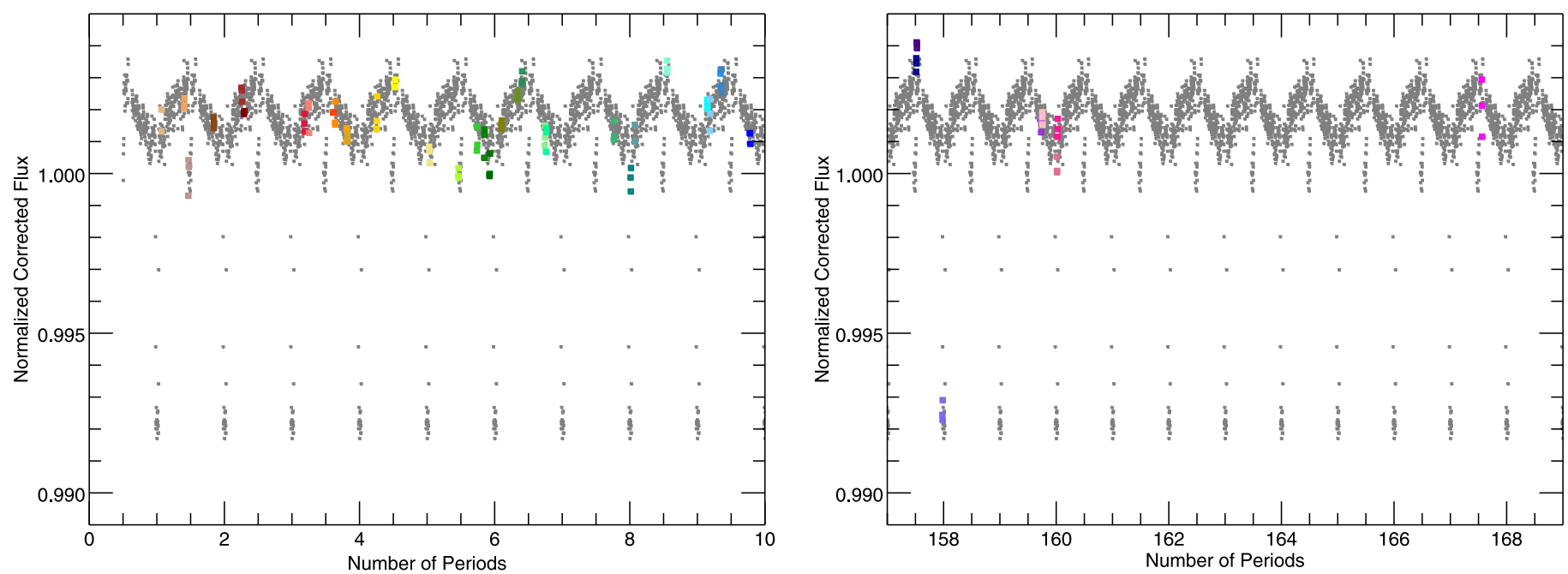

Figure 8. Flux vs. Time over the 1 year of snapshot observations. The $x$-axis is in number of periods since the first snapshot observation. Gray points are phased continuous staring mode observations repeated over many periods. Colored points are the snapshot data. Binning is the same for all data. See Section 5.3 for more details.

ipac.caltech.edu/data/SPITZER/docs/files/spitzer/preflash. txt for a discussion of the $8 \mu \mathrm{m}$ ramp). We see no evidence for a consistent ramp in our 30 minute AORs. Figure 9 shows the normalized corrected flux as a function of time for all 46 snapshot AORs binned together into 4 bins as a function of time. There is no systematic trend from the beginning to the end of the observations within $0.005 \%$, which is well below the noise level of our phase curve measured amplitude.

\section{RESULTS AND DISCUSSION}

We first examine persistent images as a remaining noise source after the above reduction. We then fit a model to our reduction of both the snapshot and continuous staring mode WASP-14b data. Next, we consider three independent metrics for judging the success of the snapshot technique. Finally, we look at the astrophysical implications of the WASP-14b data set.

\subsection{Residual Persistent images}

\subsubsection{Outliers in Snapshot Data}

The existence of persistent images under the snapshots is likely the largest uncorrected source of scatter in our snapshot data set. We examine the effect of persistent images on the snapshot observations (see Section 3.3). Our only metric for determining which of the AORs are effected by stronger latent signals is to reduce the data in two ways and then compare; first, using a superdark (theoretically no latents) and, second, using weekly darks. Out of 46 AORs, we find only three AORs whose flux densities change by more than $0.05 \%$ : the two AORs directly after the first secondary eclipse (purple and dark blue) and the AOR during transit (light purple). These three AORs were taken consecutively in time, implying that latents are the likely source of scatter in those AORs. Conversely, we can say that the remaining 43 snapshots are not affected by more than $0.05 \%$ by latent images. This is confirmed both by the small amount of scatter in the residuals (see Section Section 6.3.1) and in the difference between reductions with and without the superdark.

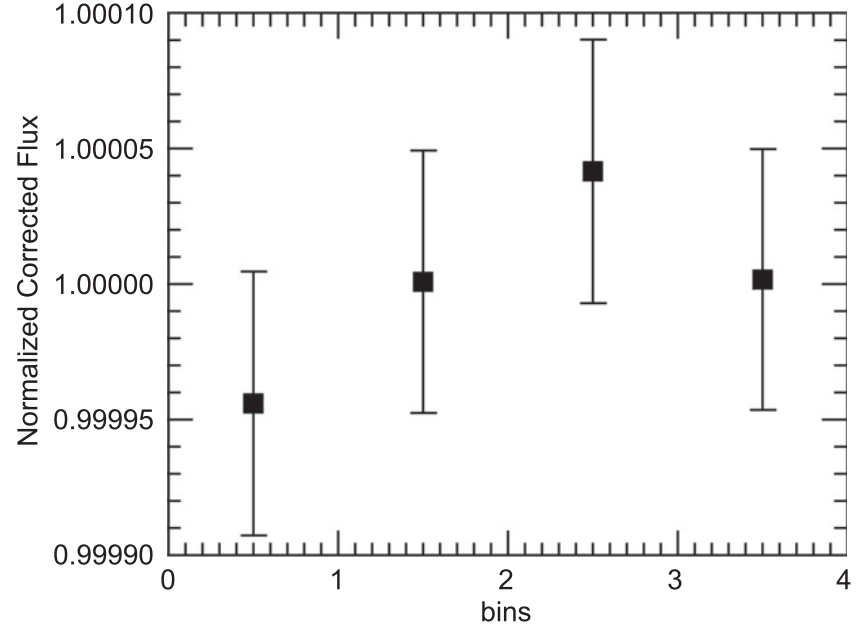

Figure 9. Corrected flux as a function of time. Fluxes for all 46 snapshot AORs have been binned together. Time is binned into four sections to look for trends as a function of time. $Y$-scale range is $0.02 \%$. There is no electronic ramp from the beginning to the end of the snapshot observations to within $50-100 \mathrm{ppm}$, which is well below our phase curve amplitude signal. See Section 5.5 for more details.

To further test if AORs with the largest delta flux density from superdark to weekly dark are indeed affected by latents, we first looked for a source of persistent images observed directly prior to the candidate high latent snapshot AORs. Approximately $2.5 \mathrm{hr}$ prior to the start of the snapshot observations, IRAC mapped the Chameleon region using a dithering and mapping strategy and not staring. This region includes some bright stars with $K=3-5$ that could cause lowlevel persistent images. Furthermore, we examined an AOR taken directly after the three snapshot AORs to look for residual persistent images. This AOR is fortunately $80 \mathrm{BCDs}$ that dither around a relatively dark portion of the sky. Median combining these images together removes all of the sources, and leaves us with an image of the background pattern that may have been present underneath our snapshot observation (see left side of Figure 10). Indeed, there are persistent images present in the median combine both from column pull-down, and 

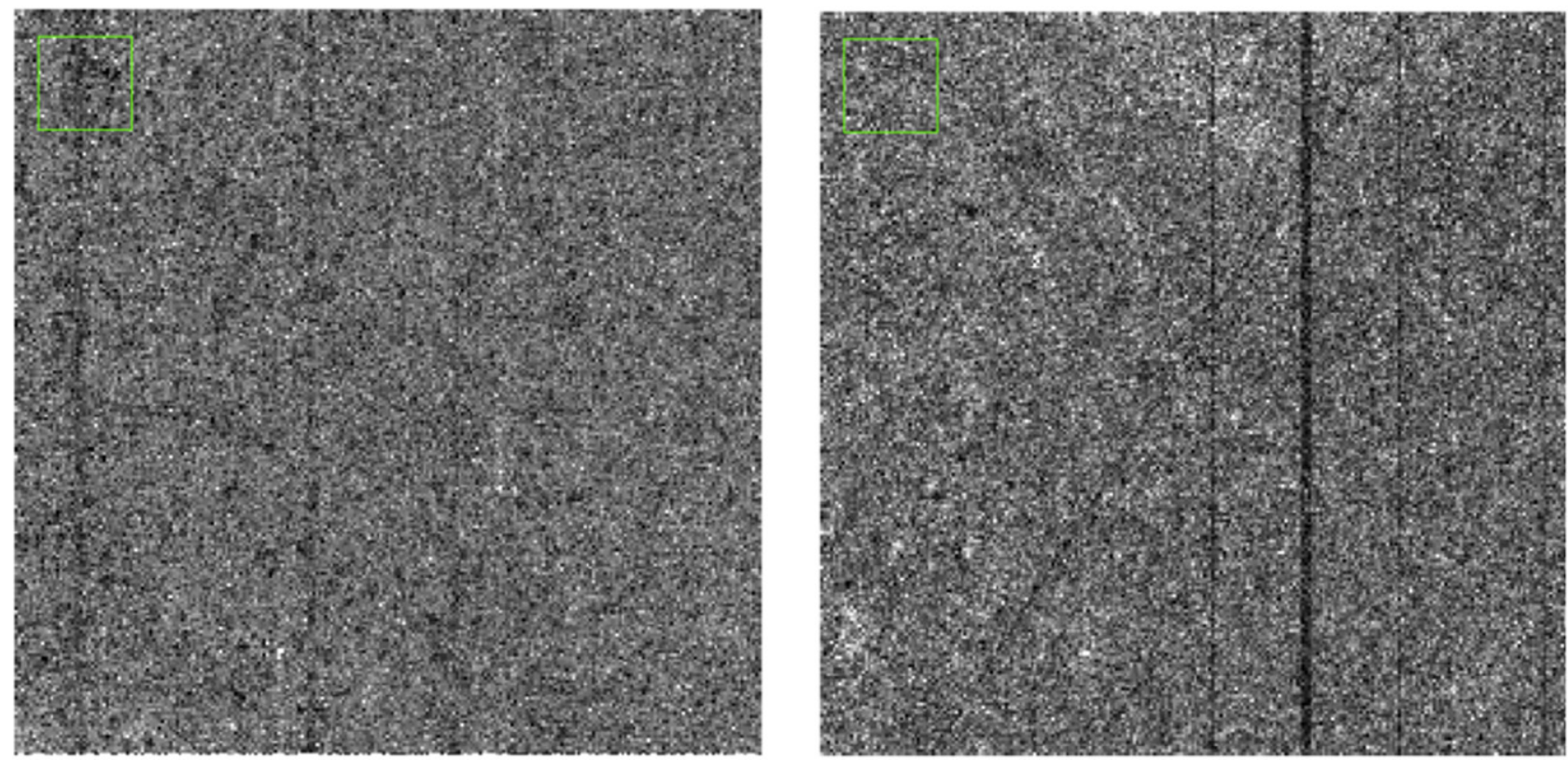

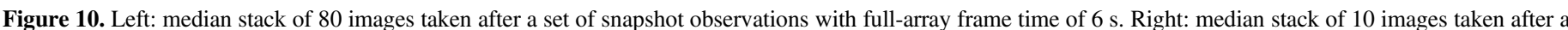

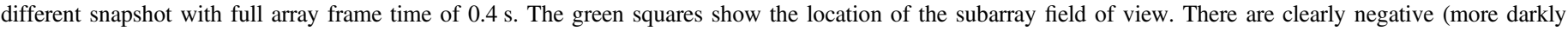

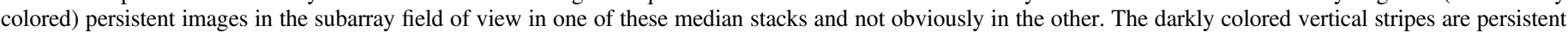

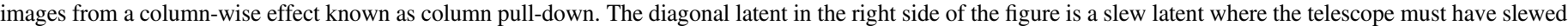
across a bright star at some time prior to these observations. See Section 6.1.1 for more details.

potentially from other bright targets all over the full array, including in the subarray region (small box in upper corner). The subarray persistent image is potentially the source of the different flux in the three snapshot observations examined here.

For comparison, we also look at median stacks of AORs observed directly after two other random snapshots (the 1st and 27th snapshots), and these show a smooth background in the subarray region, albeit with lower $\mathrm{S} / \mathrm{N}$ in the background due to less total exposure time in the median stack (see right side of Figure 10).

Not only are there long-term persistent images, as seen in the superdark analysis, but there are also short-term persistent images that decay much faster (minutes) which can affect the photometry at the $0.1 \%$ level. Additionally, it is possible that the exoplanet targets themselves are also generating persistent images, but we cannot disentangle this effect in the source photometry. Our suggestion above of dithered observations before and after each snapshot will allow us to study these types of latents and improve future observations. For the three AORs that are affected by latents, we have added $0.1 \%$ to their error bars to more accurately represent the uncertainty in flux due to persistent images.

\subsubsection{Level Offset}

One side-effect of the persistent images underneath all of our observations is that the continuous staring mode data and the snapshot data have different normalizations. We cannot know exactly what the latent behavior under these observations is, but we know that the latent behavior is different from season to season and year to year. Because this is an additive effect, we correct it by adding an offset of $0.2 \%$ uniformly to all of the snapshot data to equalize the snapshot mean flux with the continuous staring mode data (judging by eye since both sets of observations have scatter and phase curve shape). Since we are not performing absolute photometry, the additional flux offset that we manually add will make minimal difference in the conclusions of the paper.

\subsection{Phase Curve Fitting}

To test the usefulness of snapshot data sets for deriving physical parameters, we fit the Lewis et al. (2013) phase curve model to the observed phase curve of WASP-14b. This model is based on a fit of sines and cosines for the circular orbits presented in Cowan \& Agol (2008) but has been adjusted to accommodate systems with eccentricity. Given a full, continuous phase curve, there would be up to 16 fitted parameters: orbital period, inclination, $a / R_{*}$, two components of eccentricity, transit mid-point, $R_{p} / R_{*}$, depth of both secondary eclipses, four phase curve parameters, and two ramp parameters. However, since the snapshot-style observations were not designed to measure information about transits and eclipses, and those are not in fact guaranteed to be observed in any given set of snapshots, we fix 12 of those parameters and allow only the 4 phase curve shape parameters to vary. The 12 fixed parameters are fixed to the values presented in Wong et al. (2015b), listed in Table 2. The phase curve shape is defined by the following equation:

$F(\theta)=F_{0}+c 1 \cos (\theta)+c 2 \sin (\theta)+c 3 \cos (2 \theta)+c 4 \sin (2 \theta)$

,where $\mathrm{c} 1-\mathrm{c} 4$ are the free parameters, $F$ is the flux, and $\theta$ is the phase which, in the case of an eccentric orbit, is a function of the true anomaly (Lewis et al. 2013).

Figure 11 shows the snapshots and the best fits for both the continuous staring mode and snapshot data which are both reduced in exactly the same manner using the gain map. Additionally, we over-plot the fit from Wong et al. (2015b) to their reduction of the continuous staring mode data set. Data 
Table 2

Summary of Phase Curve Fitting Results

\begin{tabular}{|c|c|c|c|}
\hline Parameters & Wong et al. & pmap-continuous & pmap-snapshots \\
\hline Inclination $\left({ }^{\circ}\right)$ & 84.63 & $\ldots$ & $\ldots$ \\
\hline$a / R_{*}$ & 5.98 & $\ldots$ & $\ldots$ \\
\hline$h=e \sin (\omega)$ & -0.0792 & $\ldots$ & $\ldots$ \\
\hline$T_{0}$ & 56042.687 & $\cdots$ & $\cdots$ \\
\hline$R_{p} / R_{*}$ & 0.09421 & $\ldots$ & $\ldots$ \\
\hline Depth of 2 eclipse & 0.002367 & $\ldots$ & $\ldots$ \\
\hline $\mathrm{c} 1$ & $8.06 \mathrm{e}-04 \pm 1.3 \mathrm{e}-05$ & $7.43 \mathrm{e}-04 \pm 1.0 \mathrm{e}-06$ & $9.45 \mathrm{e}-04 \pm 2.9 \mathrm{e}-05$ \\
\hline c2 & $1.30 \mathrm{e}-04 \pm 1.7 \mathrm{e}-05$ & $2.17 \mathrm{e}-04 \pm 1.4 \mathrm{e}-06$ & $1.19 \mathrm{e}-04 \pm 3.9 \mathrm{e}-05$ \\
\hline c3 & $5.23 \mathrm{e}-05 \pm 1.5 \mathrm{e}-05$ & $2.73 \mathrm{e}-05 \pm 1.2 \mathrm{e}-06$ & $-2.09 \mathrm{e}-04 \pm 3.7 \mathrm{e}-05$ \\
\hline $\mathrm{c} 4$ & $4.51 \mathrm{e}-05 \pm 1.6 \mathrm{e}-05$ & $8.97 \mathrm{e}-05 \pm 1.3 \mathrm{e}-06$ & $-1.21 \mathrm{e}-04 \pm 3.5 \mathrm{e}-05$ \\
\hline ramp1 & 0 & $\ldots$ & $\ldots$ \\
\hline
\end{tabular}

Note. Empty values in the table indicate that those values are fixed to the Wong et al. (2015b) values. We list them here for completeness.

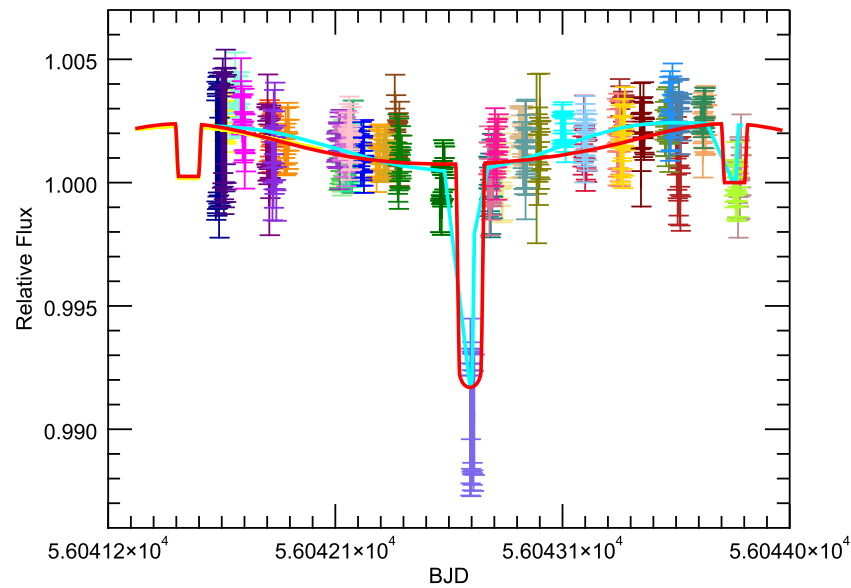

Figure 11. Fits to the snapshot and continuous staring mode data sets. Data are binned roughly by 64 photometry points. The red line is the fit from this paper to the pmap reduction of the continuous staring mode data, the cyan line is the fit to the snapshot data, and the yellow line is the Wong et al. (2015b) fit to their reduction of the continuous staring mode data (practically indistinguishable from the red line). The cyan line fit to the snapshot data is slightly irregular at transit and eclipse because there is not enough data in ingress and egress to provide a smooth fit. An extra $0.01 \%$ has been added to the error bars of all data in the AORs which we know are affected by latent images (most notably those in transit). See Section 6.2 for more details.

are shown binned at the same level at which the fitting is performed. We found the best $\chi^{2}$ values for binning at roughly the level of a set of 64 photometry points (equivalent to one fits file).

After fitting, the values of the phase curve amplitude and phase shift are derived. The uncertainties on these values are calculated by running a set of 1000 fits on a data set where the fitted parameters are randomly varied within their $1 \sigma$ error bars. The distribution of the resulting amplitudes and phase shifts is then fit with a Gaussian and the uncertainties are obtained from that Gaussian fit. Error bars in the pmap fits are likely underestimates.

Maximum flux occurs prior to phase $=0.5$ and likewise minimum flux occurs prior to phase $=0$. This is fully consistent with Wong et al. (2015b) for their continuous staring mode analysis. This implies that the hot spot is shifted eastward from the substellar point, which is found for other hot Jupiters as well (e.g., Knutson et al. 2007a, 2012; Stevenson et al. 2014; Zellem et al. 2014). General circulation models predict that the eastward hot spot implies an eastward super-rotating equatorial jet stream caused by the day to night temperature differential (Showman et al. 2015 and references therein.).

\subsubsection{Comparison with Continuous Staring Mode Data}

We compare our best-fit model for the snapshot data with two models from the continuous staring mode data. Those two models are (1) our own reduction and fit of the continuous staring mode data and (2) the independent Wong et al. (2015b) model fit to their reduction. Figure 11 shows all of the models with Wong et al. (2015b) in yellow, this paper's continuous staring mode model in red, and the snapshot model in light blue. Our model fit to our own reduction of the continuous data and the Wong et al. (2015b) fit to their reduction are extremely similar, validating this fitting technique. All three models find similar values of phase shift within the uncertainties.

The derived value of amplitude is statistically different $(\sim 5 \sigma)$ between the snapshot fit and the continuous data fits. Our fitting routine does find a slightly higher amplitude than that published in Wong et al. (2015b), but it is consistent with their value. We test whether this is a function of sampling into snapshots by subsampling the continuous data into snapshotstyle observations and fitting those observations. We do this 100 times and find a distribution of amplitudes that is consistent with our fitting of the continuous data. Therefore, we conclude that the sampling of the phase curve into snapshots is not the cause of the difference in amplitudes.

Rather than assume that there is an astrophysical source for this discrepancy, we assume instead that the uncertainties on our photometry points are underestimated because they do not include a contribution from the persistent images. Persistent images varying between snapshot observations (see Section 6.1.1) will cause the snapshot observations to vary from one epoch to another, which can cause changes to the measured 


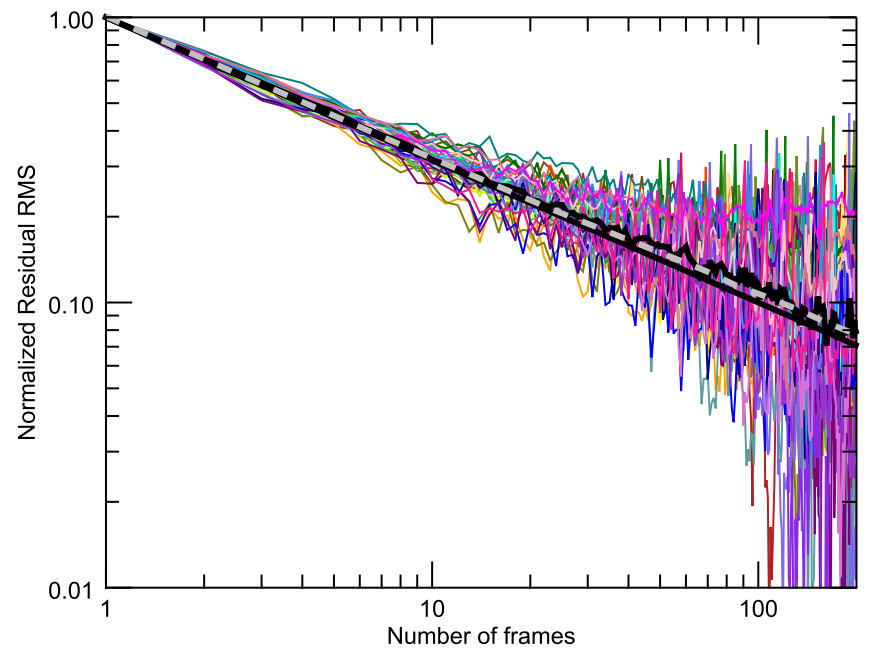

Figure 12. Normalized Residual rms vs. binning size in number of frames on the bottom and minutes on the top. Each colored line represents a single AOR. Because our observations are only 30 minutes long, the rms measurement has a lot of scatter in it at binning scales greater than a few minutes. The straight solid black line is poisson noise. The gray dashed line is the same measurement for the continuous staring mode data taken from Wong et al. (2015b). The squiggly black line is the median over all snapshots. See Section 6.3.1 for more details.

amplitude of the phase curve. Increasing the uncertainties on the snapshot photometry by 0.0001 brings the measurement of the phase amplitude in the snapshot to within the uncertainties of those measured for the continuous data set. This is consistent with the level as estimated above in our search for persistent images in some of the observations after our snapshots.

\subsection{Do Snapcurves Work?}

We present two additional pieces of evidence that the snapshot strategy can successfully obtain phase curves. First, we look for residual time-correlated noise in our snapshot observations. Second, we look at snapshot observations of a calibration star which should recover a flat light curve.

\subsubsection{Time-correlated Noise}

To test how well our reduction technique removes sources of correlated noise, we compare the rate at which binning reduces the noise to the expected rate of the square root of the number of data points for strictly poisson noise. Figure 12 shows this comparison for each of the snapshot AORs. To generate the $Y$ axis, we subtract the (fully independent) Wong et al. (2015b) model from each of our individual snapshot data, and measure the rms of the residuals. We then bin the data on increasingly large scales and plot the results as a function of the number of frames per bin. Because we only have 30 minute long AORs, these plots show significant scatter as the total number of bins per AOR gets small. The solid black line is the expected binning relation for poisson noise. The dashed gray line comes from the Wong et al. (2015b) reduction of the WASP-14b continuous staring mode data set. This continuous data set has a much longer duration and is therefore able to probe larger binning scales. The median of all of the snapshot AORs is shown by the black squiggly line and, similar to the Wong et al. (2015b) reduction, is within about $10 \%$ of the Poisson noise. For reference, our own reduction of the continuous staring mode data also follows the Wong et al. (2015b) slope.
The bright pink points at phase -0.4 in the light curve plots which appear more extended in corrected flux as a function of phase and time also diverge strongly from poisson slope in this plot (flatten out to a normalized residual rms of 0.2 by 20 binned frames). The cause for the increased scatter is unknown. The observations taken after this particular AOR are too short, and include bright stars, to determine if there could be an underlying rapidly varying latent. There is nothing obviously unusual about the position, noise pixel, or background level in that AOR as seen in Figures 4 and 6, and so we do not suspect the gain map correction.

Figure 12 shows that the nearest neighbor gain map method presented in this paper is (1) consistent with other reduction methods in that we have adequately removed the intrapixel gain effect, (2) multi-epoch data has not added new sources of correlated noise, and (3) that it is able to nearly reach the poisson noise limit for our 30 minute AORs. The heater cycling timescale for these data sets is of the order of 30-40 minutes.

\subsubsection{Snapshots of HD 158460}

Our second piece of evidence in favor of the snapshot technique comes from a secondary data set where we observed one of the IRAC calibration stars, HD 158460, with a set of 18 snapshot observations. HD 158460 was vetted by the IRAC project to be a non-variable source, and is therefore used as part of the ongoing IRAC flux calibration effort (Reach et al. 2005). It has a spectral type of $\mathrm{A} 1 \mathrm{Vn} \mathrm{C}$. The goal of this experiment is to see if our snapshot technique could recover the "truth" light curve of this non-variable calibration star, i.e., a flat line as a function of time. This test cannot be performed on any of the planet-hosting stars because they have astrophysical variability in their light curves (transits/eclipses/phase variations), whereas the IRAC calibrators have been vetted by the IRAC team to not have variations (Reach et al. 2005). Any variations from flat will indicate inconsistencies in the snapshot technique. These 18 snapshot observations were observed and reduced in exactly the same way as those of WASP-14b.

The left side of Figure 13 shows the corrected fluxes of HD 158460 as a function of time over the $\sim 200 \mathrm{hr}$ baseline of observations. Each colored data point represents an unbinned flux measurement. Black boxes show all of the data from one AOR binned together. The dashed black line is the result of a $\chi^{2}$ linear fit to the binned data points.

To test the level to which this light curve is flat, we performed a Monte Carlo simulation using 1000 instances of a Fischer-Yates shuffle to randomly switch the time stamps associated with each flux measurement, and then remeasure the slope. This shuffling must be done on the binned data points, otherwise points from one AOR would get shuffled into another AOR. The right side of Figure 13 shows a histogram of the slopes of resulting light curves from the simulation. The slope of the $\chi^{2}$ fitted linear fit to the snapshot data set is shown with a dashed line. The histogram color changes from gray to blue inside of 1 FWHM. The slope of the measured fit to the snapshot data set is within $1 \sigma$ of zero, demonstrating that the snapshot technique recovers a light curve within $1 \sigma$ of the "truth" light curve, which is a successful validation of the snapshot technique. 

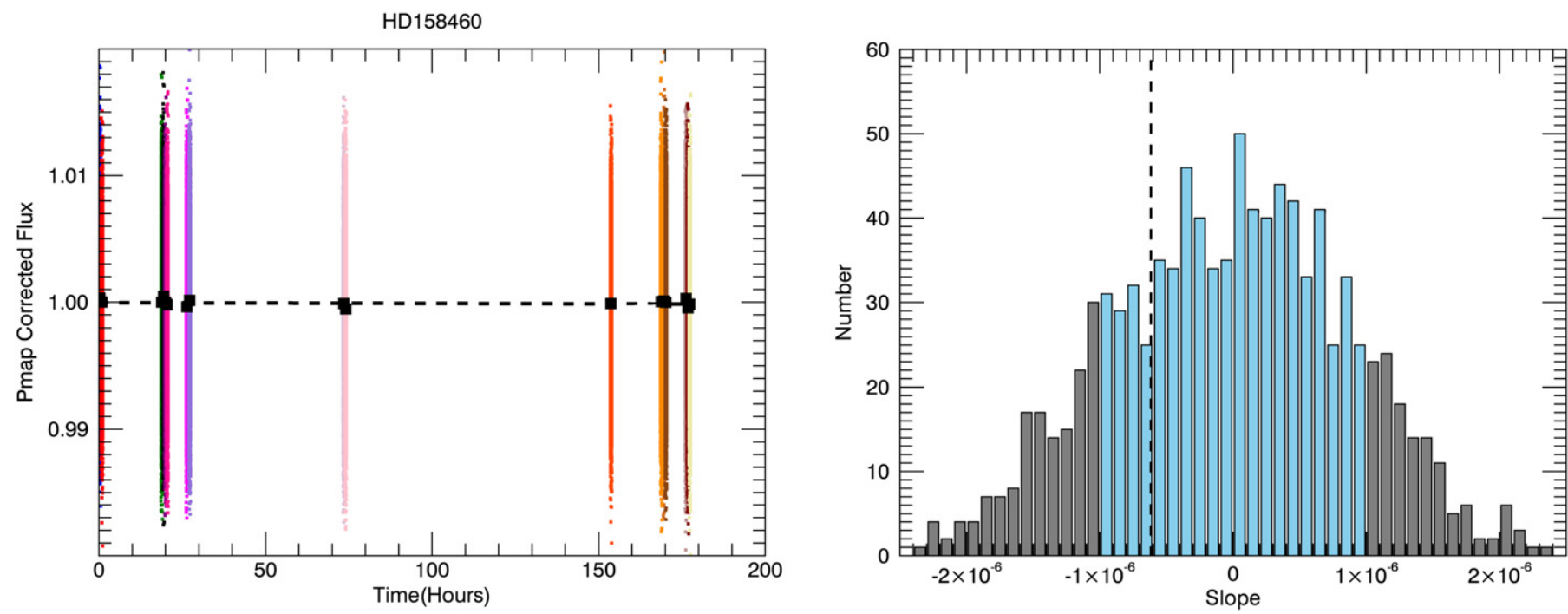

Figure 13. Snapshots of a calibration star. Left: light curve of a calibration star where each AOR is shown both unbinned with colored points and as a single binned black square. Right: a Monte Carlo simulation of possible slopes of fitted lines to this data set. The dashed line shows the chi-squared fit to the actual snapshot observations from the left plot. FWHM of a Gaussian fit to the histogram is indicated by a change in color from gray to blue. Snapshot observations are within $1 \sigma$ of a flat light curve. See Section 6.3.2 for more details.
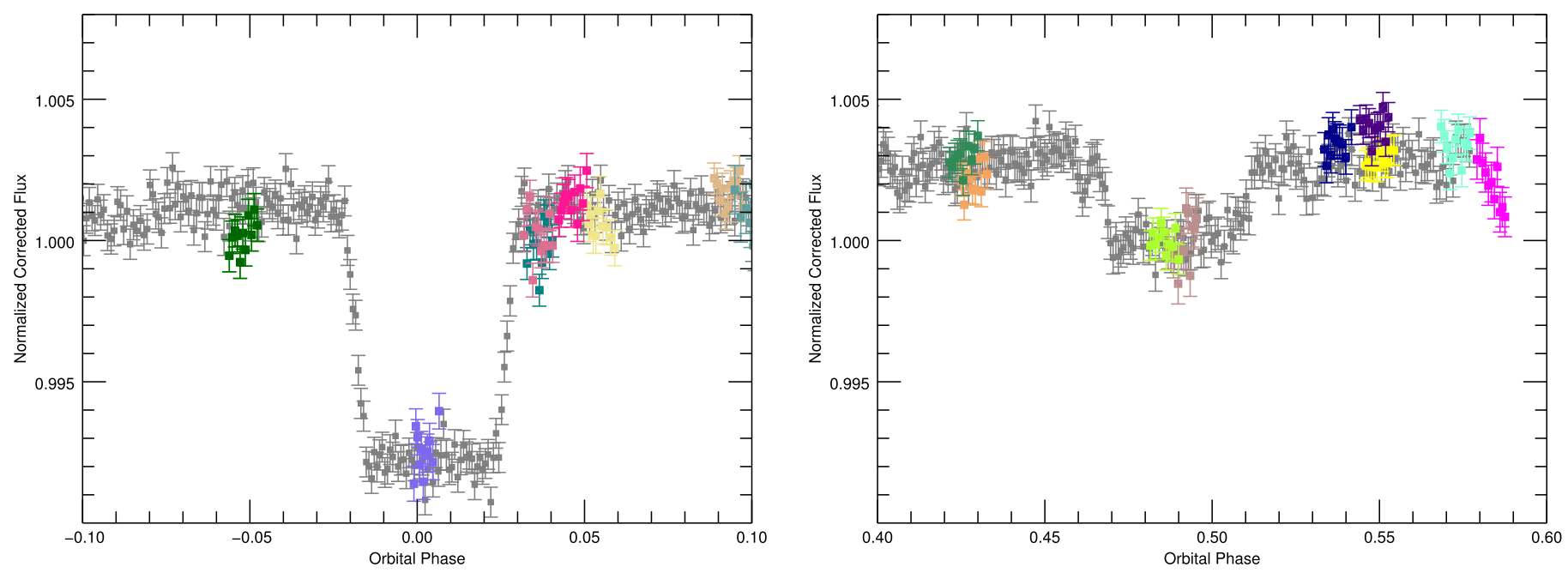

Figure 14. Comparison of the the transit (left) and secondary eclipse (right) depths between snapshot and continuous staring mode data reduced in the same way. Gray points are the continuous staring mode data, colored points are the snapshot data. Both plots have been normalized to the eclipse level. See Section 6.5 for more details.

\subsection{Phase Curve Repeatability}

Because the snapshot data set covers multiple orbits, any variation from one phase to the next can be seen in the variation between snapshots that randomly landed at similar phase in Figure 7. Astrophysical variation from phase to phase would imply that we had observed exoplanet weather across multiple periods. We find that the maximum variation between measurements (difference in average flux) at a single phase is $0.1 \%$. This implies that the maximum variation from one phase to the next is less than or equal to $0.1 \%$. The likely cause for the $0.1 \%$ variation in the residuals is the persistent images as discussed in Section 3.3, and therefore we likely have detected no significant astrophysical variation from one phase to the next. To date, there are no other descriptions of measured phase to phase variations in the literature as it is extremely difficult to disentangle the instrumental effects from astrophysical effects at such low levels. There is some discussion in theoretical works of orbit to orbit variability, predicted to be less than of the order of 1\% (Lewis et al. 2010; Kataria et al. 2013; Heng \& Showman 2015).

\subsection{Transit and Secondary Eclipse Depth}

This project was not specifically designed to measure transit or eclipse depths, however, we do have snapshot data during both transit and eclipse, and so we explore this topic briefly with the goal of seeing if we find eclipse depth variation as a function of time in the 3.5 year baseline between the first literature measurements and this paper. Figure 14 shows a zoom-in for a comparison of our snapshot and continuous staring mode data at both transit (left) and eclipse (right). Our snapshot transit point is one of those affected by the latent as noted in the superdark analysis, and so while our value is consistent with that in the literature, we make no comment on the change in transit depth as a function of time. 
We measure an eclipse depth (with two snapshots in eclipse) of $0.222 \pm 0.07 \%$. The continuous staring mode data includes two secondary eclipses and Wong et al. (2015b) measure the depths to be $0.2115_{-.0114}^{+.0135} \%$ and $0.2367_{-0.0142}^{+0.009} \%$. From a secondary eclipse observation in 2009 March (3-3.5 years prior to the observations presented here), Blecic et al. (2013) measure the depth of the secondary to be $0.224 \pm 0.018 \%$. Our measured eclipse depth is consistent with the range presented in those papers, and so we see no evidence for an eclipse depth change as a function of time for WASP-14b.

\section{CONCLUSION}

In summary, we describe Spitzer IRAC snapshot highprecision photometry of WASP-14b. We describe a new reduction technique which is now provided for public use on the Spitzer Science Center website in which we calibrate out the shape of the intrapixel gain using a well-studied flat light curve star. We show three pieces of evidence that this technique is effective in measuring phase curves in a more efficient manner than with continuous staring mode data. Conclusions specific to WASP-14b are that we confirm an eastward hot spot with an amplitude of the phase curve between 0.00078 and 0.00098 . We place limits on the phase to phase astrophysical variation of the system at less than $0.1 \%$, although that is probably an overestimate and due mainly to instrumental systematics. Finally, we see no evidence for a change in eclipse depth over a 3.5 year baseline from archival observations to our most recent observations.

The largest limitation of this new technique is the effect of latent images caused by previous observations on the stability of the photometry. For future snapshot observations, we recommend observing a dither pattern of a blank field after each snapshot to check for the existence of persistent images and hopefully to remove that "dark pattern" from the data. To be even more safe, it may be advantageous to observe all of the snapshots in the same visibility window so that we are not dealing with very different low-level latents. Of course, there can always be local in-time latents, but the first recommendation should help with understanding those patterns.

In this paper, it was useful to have a snapshot observation in transit and in eclipse, mostly as checks on the phase curve shape. For this data set, it happened randomly but fortuitously. In the future, it might therefore be useful to specifically schedule an observation both at transit and eclipse.

We thank the referee Nikole Lewis for useful suggestions which have greatly improved the manuscript. This research has made use of data from the Infrared Processing and Analysis Center/California Institute of Technology, funded by the National Aeronautics and Space Administration and the National Science Foundation. This work was based on observations obtained with the Spitzer Space Telescope, which is operated by the Jet Propulsion Laboratory, California Institute of Technology under a contract with NASA. This research has made use of the NASA Exoplanet Archive, which is operated by the California Institute of Technology, under contract with the National Aeronautics and Space Administration under the Exoplanet Exploration Program. This research has made use of the NASA/ IPAC Infrared Science Archive, which is operated by the Jet Propulsion Laboratory, California Institute of Technology, under contract with the National Aeronautics and Space Administration. This research has made use of the SIMBAD database, operated at CDS, Strasbourg, France. This research has made use of exoplanet.eu.

Facility: Spitzer(IRAC).

\section{REFERENCES}

Ballard, S., Charbonneau, D., Deming, D., et al. 2010, PASP, 122, 1341 Blecic, J., Harrington, J., Madhusudhan, N., et al. 2013, ApJ, 779, 5 Burrows, A., Budaj, J., \& Hubeny, I. 2008, ApJ, 678, 1436

Burrows, A., Rauscher, E., Spiegel, D. S., \& Menou, K. 2010, ApJ, 719, 341

Burrows, A., Sudarsky, D., \& Hubeny, I. 2006, ApJ, 650, 1140

Campo, C. J., Harrington, J., Hardy, R. A., et al. 2011, ApJ, 727, 125

Charbonneau, D., Allen, L. E., Megeath, S. T., et al. 2005, ApJ, 626, 523

Ciardi, D. R., von Braun, K., Bryden, G., et al. 2011, AJ, 141, 108

Cooper, C. S., \& Showman, A. P. 2005, ApJL, 629, L45

Cowan, N. B., \& Agol, E. 2008, ApJL, 678, L129

Cowan, N. B., \& Agol, E. 2011, ApJ, 726, 82

Cowan, N. B., Agol, E., \& Charbonneau, D. 2007, MNRAS, 379, 641

Cowan, N. B., Machalek, P., Croll, B., et al. 2012, ApJ, 747, 82

Crossfield, I. J. M., Barman, T., Hansen, B. M. S., Tanaka, I., \& Kodama, T. 2012a, ApJ, 760, 140

Crossfield, I. J. M., Hansen, B. M. S., Harrington, J., et al. 2010, ApJ, 723,1436

Crossfield, I. J. M., Knutson, H., Fortney, J., et al. 2012b, ApJ, 752, 81

Deming, D., Knutson, H., Agol, E., et al. 2011, ApJ, 726, 95

Deming, D., Knutson, H., Kammer, J., et al. 2015, ApJ, 805, 132

Deming, D., Seager, S., Richardson, L. J., \& Harrington, J. 2005, Natur, 434, 740

Demory, B.-O., de Wit, J., Lewis, N., et al. 2013, ApJL, 776, L25

Ehrenreich, D., \& Désert, J.-M. 2011, A\&A, 529, A136

Fazio, G. G., Hora, J. L., Allen, L. E., et al. 2004, ApJS, 154, 10

Fortney, J. J., Cooper, C. S., Showman, A. P., Marley, M. S., \& Freedman, R. S. 2006, ApJ, 652, 746

Fortney, J. J., Lodders, K., Marley, M. S., \& Freedman, R. S. 2008, ApJ, 678, 1419

Grillmair, C. J., Burrows, A., Charbonneau, D., et al. 2008, Natur, 456, 767

Harrington, J., Hansen, B. M., Luszcz, S. H., et al. 2006, Sci, 314, 623

Heng, K., \& Showman, A. P. 2015, AREPS, 43, 509

Ingalls, J. G., Krick, J. E., Carey, S. J., et al. 2012, Proc. SPIE, 8442, 84421 Y

Ingalls, J. G., Krick, J. E., Carey, S. J., et al. 2016, AJ, in press (arXiv: 1601.05101)

Johnson, J. A., Winn, J. N., Albrecht, S., et al. 2009, PASP, 121, 1104

Joshi, Y. C., Pollacco, D., Collier Cameron, A., et al. 2009, MNRAS, 392,1532

Kataria, T., Showman, A. P., Lewis, N. K., et al. 2013, ApJ, 767, 76

Knutson, H. A., Charbonneau, D., Allen, L. E., et al. 2007a, Natur, 447, 183

Knutson, H. A., Charbonneau, D., Burrows, A., O’Donovan, F. T., \& Mandushev, G. 2009a, ApJ, 691, 866

Knutson, H. A., Charbonneau, D., Cowan, N. B., et al. 2009b, ApJ, 690, 822 Knutson, H. A., Charbonneau, D., Cowan, N. B., et al. 2009c, ApJ, 703, 769 Knutson, H. A., Fulton, B. J., Montet, B. T., et al. 2014, ApJ, 785, 126 Knutson, H. A., Howard, A. W., \& Isaacson, H. 2010, ApJ, 720, 1569 Knutson, H. A., Lewis, N., Fortney, J. J., et al. 2012, ApJ, 754, 22

Krick, J., Ingalls, J., Carey, S., et al. 2015, IRAC High Precision Photometry Website, http://irachpp.spitzer.caltech.edu

Krick, J. E., Glaccum, W. J., Carey, S. J., et al. 2012, ApJ, 754, 53

Krick, J. E., Surace, J. A., Thompson, D., et al. 2009, ApJS, 185, 85

Laughlin, G., Deming, D., Langton, J., et al. 2009, Natur, 457, 562

Lewis, N. K., Knutson, H. A., Showman, A. P., et al. 2013, ApJ, 766, 95

Lewis, N. K., Showman, A. P., Fortney, J. J., et al. 2010, ApJ, 720, 344

Madhusudhan, N. 2012, ApJ, 758, 36

Maxted, P. F. L., Anderson, D. R., Doyle, A. P., et al. 2013, MNRAS, 428, 2645

Menou, K., \& Rauscher, E. 2010, ApJ, 713, 1174

Mighell, K. J. 2005, MNRAS, 361, 861

Nadaraya, E. A. 1964, Theory of Probability and Its Applications, 9, 141

Paulson, D. B., Cochran, W. D., \& Hatzes, A. P. 2004, AJ, 127, 3579

Perez-Becker, D., \& Showman, A. P. 2013, ApJ, 776, 134

Reach, W. T., Megeath, S. T., Cohen, M., et al. 2005, PASP, 117, 978

Santos, N. C., Mayor, M., Naef, D., et al. 2000, A\&A, 361, 265

Schwartz, J. C., \& Cowan, N. B. 2015, MNRAS, 449, 4192

Showman, A. P., Lewis, N. K., \& Fortney, J. J. 2015, ApJ, 801, 95

Sing, D. K., \& López-Morales, M. 2009, A\&A, 493, L31

Spiegel, D. S., Silverio, K., \& Burrows, A. 2009, ApJ, 699, 1487

Stevenson, K. B., Désert, J.-M., Line, M. R., et al. 2014, Sci, 346, 838 
Stevenson, K. B., Harrington, J., Fortney, J. J., et al. 2012, ApJ, 754, 136 Thrastarson, H. T., \& Cho, J. Y. 2010, ApJ, 716, 144

Todorov, K. O., Deming, D., Knutson, H. A., et al. 2012, ApJ, 746, 111

Todorov, K. O., Deming, D., Knutson, H. A., et al. 2013, ApJ, 770, 102

Watson, G. S. 1964, Sankhyā: The Indian Journal of Statistics, Series A, 26,359
Werner, M. W., Roellig, T. L., Low, F. J., et al. 2004, ApJS, 154, 1 Wong, I., Knutson, H. A., Kataria, T., et al. 2015a, arXiv:1512.09342 Wong, I., Knutson, H. A., Lewis, N. K., et al. 2015b, ApJ, 811, 122 Zellem, R. T., Lewis, N. K., Knutson, H. A., et al. 2014, ApJ, 790, 53 Zhao, M., Monnier, J. D., Swain, M. R., Barman, T., \& Hinkley, S. 2012, ApJ, 744,122 